OU-HET 725/2011

\title{
QCD parity violation in a quarkonium via SUSY
}

\author{
Naoyuki Haba, Kunio Kaneta, and Tetsuya Onogi \\ Department of Physics, Osaka University, Toyonaka, Osaka 560-0043, Japan
}

\begin{abstract}
The supersymmetric standard model undergoes parity violation in QCD through chiral quark-squark-gluino interactions with non-degenerate masses between left-handed and right-handed squarks. Since experiments have not shown any parity violation in QCD yet, a bound for the mass degeneracy between left-handed and right-handed squarks should exist. In this paper we try to obtain this bound for each squark. Firstly, we investigate a non-degeneracy bound between $m_{\tilde{c}_{L}}$ and $m_{\tilde{c}_{R}}$ from experimental data of charmonium decay. Secondly, we estimate the non-degeneracy bounds for $\tilde{u}$ and $\tilde{d}$ from nucleon-meson scattering data, and comment on other squarks.
\end{abstract}

Keywords: QCD; NRQCD; SUSY; parity violation; quarkonium; squark mass 


\section{Introduction}

The supersymmetric (SUSY) standard model (SM) is one of the most promising candidates beyond the SM due to gauge coupling unification, the possible existence of dark matter, and so on. It is worth noting that the SUSY SM has chiral gauge interactions in fermion-sfermiongaugino vertexes, for example, left-handed fermions only couple to left-handed sfermions with gauginos. The left-right index in sfermions is just a label and has nothing to do with spin, however, fermion-sfermion-gaugino interactions are exactly chiral. Moreover, the mass of the left-handed sfermion is not the same as that of the right-handed sfermion in general, and this non-degeneracy is also induced from radiative corrections picking up weak interactions. Therefore, due to this non-degeneracy, SUSY gauge interactions cause parity violation even in QCD! This is a remarkable feature of the SUSY SM, and this effect is negligible in a lot of other candidates beyond the SM[1]. We take a setup of $R$-parity conservation, where sparticles propagate only inside loop diagrams due to their heavy masses. Since any parity violation has not been discovered in QCD, non-degeneracy bounds should be obtained between the leftand right-handed squark masses.

Does the experimental fact of conservation of parity in QCD suggest a degeneracy between left- and right-handed squarks? We must investigate this degeneracy, and try to obtain nondegeneracy bounds from current experimental data. As for the degeneracy of $\tilde{t}_{L}$ and $\tilde{t}_{R}$, there have been some research on the process of $t \bar{t}$ pair production in collider experiments [1, 2]. Amplitudes of $t \bar{t}$ pair production were found their depend on helicities,and non-degeneracy between $\tilde{t}_{L}$ and $\tilde{t}_{R}$ causes the asymmetry measurement of the cross section. The case of $\mathcal{O}(100)$ $\mathrm{GeV}(\mathcal{O}(1) \mathrm{TeV})$ masses of sparticles was investigated in Ref. [2] (Ref. [1]).

Here we should comment on sparticle masses which are consistent with collider experiments. One case is that all sparticles are heavy of $\mathcal{O}(1) \mathrm{TeV}$ as well as gluino mass $>600 \mathrm{GeV}[3]$, where all sparticles are too heavy to be detected at detectors in current experiments. Another case is that light sparticles exist which are degenerate to $30 \mathrm{GeV}$ compared to other heavy sparticles. It is because there are experimental cuts for $p_{T} \mathrm{~s}$ multi-jets with missing transverse momentum in the SUSY search at the LHC (Tevatron), where an event selection for jets is $p_{T}>40 \mathrm{GeV}$ [3] $\left(p_{T}>30 \mathrm{GeV}[4]\right)$, and $p_{T}$ of jets are roughly estimated as the mass difference of gluino and squarks. Thus, the degeneracy of sparticles within $30 \mathrm{GeV}$ is consistent with collider experiments. We will consider both cases in the following numerical analyses.

In this paper, we try to obtain the bound for left-right degeneracy of squark masses other than the stop. At first, we investigate a non-degeneracy bound between $m_{\tilde{c}_{L}}$ and $m_{\tilde{c}_{R}}$ from experimental data of charmonium decay. For this analys, we use non-relativistic QCD (NRQCD) [5], since the charmonium is heavy. The $q \bar{q}$ bound state in NRQCD is considered in Section 2, and some related calculations are shown in Appendix A. We utilize dimension six operators to evaluate a non-degeneracy bound. Dimension six operators are discussed in Appendix B. 
In Section 3 and 4, we calculate a formula for the decay width by using an effective action technique. In Section 5, we estimate bounds for left-right non-degeneracy of $\tilde{c}$. We also estimate the non-degeneracy bounds for $\tilde{u}$ and $\tilde{d}$ from nucleon-meson scattering data, and finally comment on bounds for other squarks.

\section{Quarkonium in NRQCD}

Let us consider a quarkonium of $q \bar{q}$ bound state in the NRQCD framework by introducing a bilocal field. It is applicable for heavy quarks, and a related work has been shown in, for example, Refs. [6, 7, 8, 9, 10, 11, 12].

A two-body effective action in NRQCD is given by

$$
S_{\text {eff }}=\int_{X} \int_{\vec{r}} \phi_{X}^{\mu \dagger}(\vec{r})\left[i \partial_{X}^{0}-\frac{\nabla_{X}^{2}}{4 m}+H(r)\right] \phi_{\mu X}(\vec{r}),
$$

where $H(r)$ is defined as $H(r) \equiv-\nabla_{r}^{2} / m-V(r)$. A detail derivation of Eq. (2.1) is given in Appendix A. Now we estimate the spectra of bound states $\phi_{X}^{\mu}(\vec{r}) . \phi_{X}^{\mu}(\vec{r})$ can be expanded by a complete set of $\psi_{n}(\vec{r})$ as

$$
\phi_{X}^{\mu}(\vec{r})=\sum_{n} a_{n}^{\mu}(X) \psi_{n}(\vec{r})=\sum_{n} \int \frac{d^{3} P}{(2 \pi)^{3}} a_{n}^{\mu}(\vec{P}) \psi_{n}(\vec{r}) e^{-i P \cdot X},
$$

where $a_{n}^{\mu}(X)$ is a plane wave, and $\psi_{n}(\vec{r})$ is a possible bound state which this system can take. An eigenstate of $H(\vec{r})$, which satisfies

$$
\hat{H}(\hat{\vec{r}}) \psi_{n}(\vec{r})=E_{n} \psi_{n}(\vec{r})
$$

is a quarkonium, and $E_{n}$ denotes a binding energy of it. Orthogonality and completness suggest

$$
\int d^{3} r \psi_{n}^{\dagger}(\vec{r}) \psi_{m}(\vec{r})=\delta_{n m}, \quad \sum_{n} \psi_{n}(\vec{r}) \psi_{n}^{\dagger}(\vec{s})=\delta(\vec{r}-\vec{s}) .
$$

A hadron wave function is factorized by $a_{n}^{\mu}(X)$, which only depends on center of mass coordinate. Here $\mu$ represents spin singlet (triplet) state of meson when $\mu=0(\mu=i)$. Note that a hadron labeled by $n$ is created by $a_{n}^{\mu \dagger}(X)$ as $a_{n}^{\mu \dagger}(X)|0\rangle=|n\rangle$.

Here let us apply this formalism to a charmonium, for example. We denote $n=\eta_{c}, h_{c}, J / \psi$, $\chi_{c}, \cdots$, then a spin singlet state $\phi_{X}^{0}(\vec{r})$ and a spin triplet state $\phi_{X}^{i}(\vec{r})$ are represented by

$$
\begin{aligned}
& \phi_{X}^{0}(\vec{r})=a_{\eta_{c}}^{0}(X) \psi_{\eta_{c}}(\vec{r})+a_{h_{c}}^{0}(X) \psi_{h_{c}}(\vec{r})+\cdots, \\
& \phi_{X}^{i}(\vec{r})=a_{J / \psi}^{i}(X) \psi_{J / \psi}(\vec{r})+a_{\chi_{c J}}^{i}(X) \psi_{\chi_{c J}}(\vec{r})+\cdots,
\end{aligned}
$$

respectively. We now obtain the effective action of charmonium in the SM QCD, where parity is conserved. 


\section{Direct parity violation}

In the SUSY SM, parity can be violated in quarkonium through the non-degeneracy of left-right squark masses. As we have shown in Appendix B, there are three parity-violating operators, $\mathcal{O}_{4 F}^{(1)}, \mathcal{O}_{4 F}^{(8)}$, and $\mathcal{O}_{q q G}$. At a direct decay vertex of quarkonium, $\mathcal{O}_{4 F}^{(1)}$ gives the leading order of parity violation, and we call this process "direct parity violation". The explicit form of the direct parity violating operator is given by

$$
\mathcal{O}_{4 F}^{\mathrm{p} \cdot \mathrm{v}}=\left(A_{u c}+B_{c u}\right) \delta^{4}(x-y)\left[\bar{u}(x) \gamma_{\mu} u(y)\right]\left[\bar{c}(x) \gamma^{\mu} \gamma^{5} c(y)\right]
$$

where $A_{u c}$ and $B_{c u}$ are

$$
\begin{aligned}
& A_{u c} \equiv \frac{12 g_{s}^{4}}{192 \pi^{2}} \frac{1}{4}\left(-C_{L L}^{(\tilde{u}, \tilde{c})}+C_{R R}^{(\tilde{u}, \tilde{c})}+C_{L R}^{(\tilde{u}, \tilde{c})}-C_{R L}^{(\tilde{u}, \tilde{c})}\right), \\
& B_{c u} \equiv \frac{12 g_{s}^{4}}{192 \pi^{2}} \frac{1}{4}\left(-C_{L L}^{(\tilde{c}, \tilde{u})}+C_{R R}^{(\tilde{c}, \tilde{u})}-C_{L R}^{(\tilde{c}, \tilde{u})}+C_{R L}^{(\tilde{c}, \tilde{u})}\right),
\end{aligned}
$$

respectively. We estimate $u$-quark contribution at first, and later include $d$-quark contribution. Note that squark flavor is labeled by $C_{i j}^{\left(\tilde{q}, \tilde{q}^{\prime}\right)}(i, j=L, R)$, and has squark mass dependence through $f_{1}\left(m_{\tilde{q}}, m_{\tilde{q}^{\prime}}\right)$ and $f_{2}\left(m_{\tilde{q}}, m_{\tilde{q}^{\prime}}\right)$. For example, $C_{L L}^{(\tilde{u}, \tilde{c})}$ is denoted as

$$
C_{L L}^{(\tilde{u}, \tilde{c})}=\frac{2}{9}\left[f_{1}\left(m_{\tilde{u}_{L}}, m_{\tilde{c}_{L}}\right)+f_{2}\left(m_{\tilde{u}_{L}}, m_{\tilde{c}_{L}}\right)\right],
$$

and other $C$-factors are similarly obtained by using Eqs. $(\underline{B .3}) \sim(\bar{B} .6)$.

As for $\mathcal{O}_{q q G}$ and $\mathcal{O}_{4 F}^{(8)}$, they do not induce the leading order contributions, because they must emit a gluon in the decay vertex. We can neglect gluon exchange between in-going and out-going states at the decay instant in the NRQCD, since non-relativistic bound states are hadronized by space-like gluon exchanges. Therefore, we can neglect the contributions from $\mathcal{O}_{q q G}$ and $\mathcal{O}_{4 F}^{(8)}$, and factorize this decay process by a vacuum insertion as in Fig. 1.

We focus on a charmonium, $\eta_{c}$, which is $0^{-+}$under $J^{P C}$, and has mass of $2980 \mathrm{MeV}$. Notice that $\mathcal{O}_{4 F}^{\text {p.v }}$ is a contact interaction, where the decay constant is a value of wave function at an origin due to $\delta$-function and a decay through the contact interaction is only possible with the S-state (angular momentum $L=0$ ). Thus, reminding $\pi$ is $0^{-+}, \eta_{c}$ can not decay to $\pi \pi$ until it pick up parity violation, since $\pi(p), \pi(-p)$ system* of S-state is $0^{++}$. Note that there exits weak interaction, however, it also breaks $C$. Anyhow, as in Fig. 1, the direct parity violation through the SUSY effects, i.e., a two-body decay process, $\eta_{c} \rightarrow \pi \pi$, should be factorized as $\left\langle\pi \pi\left|\mathcal{O}_{4 F}^{\text {p.v }}\right| \eta_{c}\right\rangle \sim\left\langle\pi \pi\left|\bar{q} \gamma^{\mu} q\right| 0\right\rangle\left\langle 0\left|\bar{q} \gamma_{\mu} \gamma^{5} q\right| \eta_{c}\right\rangle$. Here $\left\langle\pi \pi\left|\bar{q} \gamma^{\mu} q\right| 0\right\rangle$ is a pion form factor, and we can estimate $\left\langle 0\left|\bar{q} \gamma_{\mu} \gamma^{5} q\right| \eta_{c}\right\rangle$ by use of NRQCD. Actually, by regarding $\bar{q} \gamma^{0} \gamma^{5} q \sim-\frac{1}{2} \chi^{\dagger} \varphi+$ h.c. in a non-relativistic picture, the S-matrix element of $\eta_{c} \rightarrow \pi \pi$ is given by

$$
\left\langle\pi \pi\left|\mathcal{O}_{4 F}^{\mathrm{p} . \mathrm{v}}\right| \eta_{c}\right\rangle \sim-\frac{1}{2}\left(A_{u c}+B_{c u}\right) \delta^{4}(x-y)\left\langle\pi \pi\left|u^{\dagger}(x) u(y)\right| 0\right\rangle\left\langle 0\left|\chi^{\dagger}(x) \varphi(y)\right| \eta_{c}\right\rangle .
$$

* It has $P=(-1)^{L}$ and $C=(-1)^{S+L}$. 


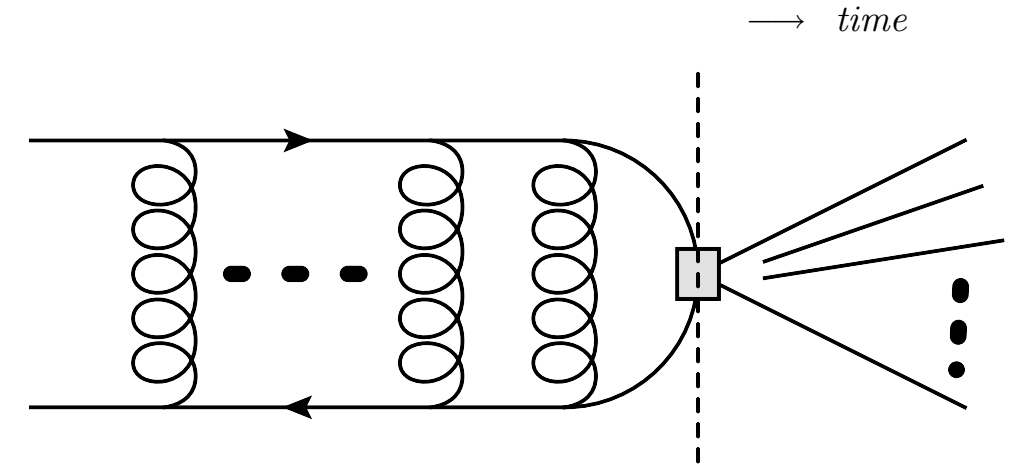

Figure 1: Factorization by a vacuum insertion in a direct parity violation process. A box stands for $\mathcal{O}_{4 F}^{\text {p.v }}$ in Eq. (3.1).

Here $\left\langle\pi \pi\left|u^{\dagger}(x) u(y)\right| 0\right\rangle \sim F^{s}(k)$ is a scalar form factor of pion, which has non-trivial energy dependence.

In general, when a bound state $|n\rangle\left(\equiv a_{n}^{\nu \dagger}(P)|0\rangle\right)$ decays through a bilocal operator $\mathcal{O}_{X}^{\nu \lambda \cdots}(\vec{r})=$ $\phi_{X}^{\mu}(\vec{r}) \phi_{X}^{\lambda}(\vec{r}) \cdots$, its matrix element is given by

$$
\left\langle 0\left|T\left[\mathcal{O}_{X}^{\nu \lambda \cdots}(\vec{r})\right]\right| n\right\rangle=i \int d^{4} Y \int d^{3} s F_{P}^{n}(Y ; s)\left(i \partial_{Y^{0}}-\frac{\nabla_{Y}^{2}}{4 m}-\hat{H}(s)\right)\left\langle 0\left|T\left[\mathcal{O}_{X}^{\nu \lambda \cdots}(\vec{r}) \phi_{Y}^{\mu \dagger}(s)\right]\right| 0\right\rangle,
$$

where $F_{P}^{n}(Y ; s) \equiv \psi_{n}(\vec{s}) e^{-i P \cdot Y}$, and it satisfies $\int d^{3} X d^{3} r \phi_{X}^{\mu \dagger}(r) F_{P}^{n}(X ; r)=a_{n}^{\dagger}(\vec{P})$ from orthogonality and completeness. Thus, the transition amplitude in Eq.(3.6) is given by $\psi_{n}(\vec{r}) e^{-i P X}$ with $\mathcal{O}_{X}^{\nu \lambda \cdots}(\vec{r})=\phi_{X}^{\nu}(\vec{r})$.

Let us go back to a charmonium, and take $q$ as $c$-quark in Eq.(A.3). Since a heavy quark is non-relativistically expanded as Eq. (A.3), the 4-Fermi operator can be also expanded similarly. In the leading order, components of $\chi^{\dagger} \varphi$ and $\varphi^{\dagger} \chi$ in the bilocal field, are only creating and annihilating operators of charmonium. Thus, $\phi_{X}^{n}(\vec{r})$ corresponds to $\chi^{\dagger}(x) \varphi(y)$, and we name a label $n=0 \eta_{c}$ for the charmonium, which suggests

$$
\left\langle 0\left|\phi_{X}^{0}(\vec{r})\right| \eta_{c}\right\rangle=\psi_{\eta_{c}}(\vec{r}) e^{-i P \cdot X} .
$$

Remind that $\mathcal{O}_{4 F}^{\text {p.v }}$ is a contact interaction, and we can use $m_{\eta_{c}}$ for an energy of the pion form factor due to a momentum conservation. Then, we obtain

$$
\left\langle\pi \pi\left|\mathcal{O}_{4 F}^{\text {p.v }}\right| \eta_{c}\right\rangle \sim-\frac{1}{2}\left(A_{u c}+B_{c u}\right) F^{s}\left(m_{\eta_{c}}\right) \psi_{\eta_{c}}(0) .
$$

There is a $d$-quark contribution as well as $u$-quark ones, so that the effective 4-Fermi operator $\mathcal{O}_{4 F}^{\text {p.v }}$ becomes a linear combination of $u$ and $d$. Therefore, $\Gamma\left(\eta_{c} \rightarrow \pi \pi\right)$ is estimated as

$$
\Gamma\left(\eta_{c} \rightarrow \pi \pi\right) \sim\left|A_{u c}+A_{d c}+B_{c u}+B_{c d}\right|^{2} \frac{\left|F^{s}\left(m_{\eta_{c}}\right)\right|^{2}\left|\psi_{\eta_{c}}(0)\right|^{2}}{16 m_{\eta_{c}}^{2}} .
$$


Since $\eta_{c}$ is an S-state, the decay width depends only on the wave function at the origin . This is a characteristic feature in the direct parity violating process in the SUSY SM.

\section{Indirect parity violation}

The QCD dimension six operators from the SUSY SM can have the parity violating effects, and actually, they can also contribute organization of quarkoniums themselves. We call this effect "indirect parity violation", and we investigate it in this section. For this indirect parity violation, all $\mathcal{O}_{q q G}, \mathcal{O}_{4 F}^{(1)}$, and $\mathcal{O}_{4 F}^{(8)}$ contribute as in Fig. 2.
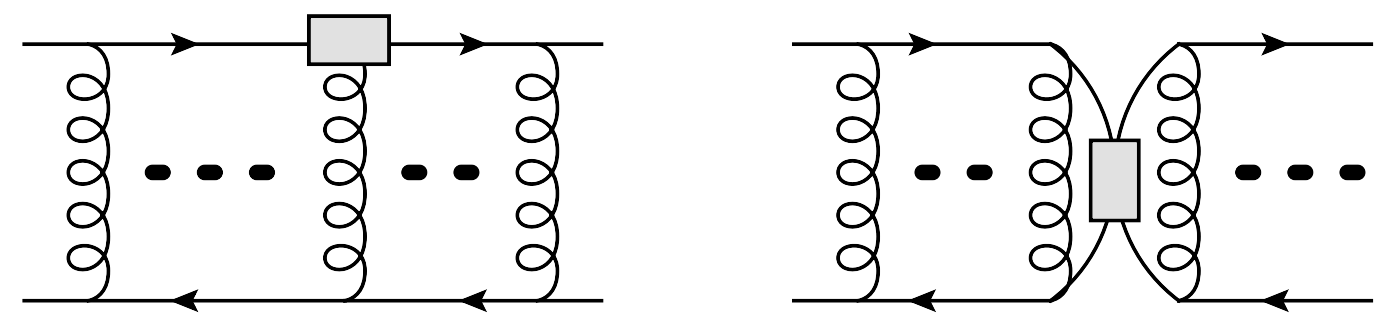

Figure 2: Diagrams which contribute indirect parity violation through dimension six operators (which are shown a box at a vertex). (Left): a contribution from $\mathcal{O}_{q q G}$, (Right): a contribution from $\mathcal{O}_{4 F}^{(1)}$ or $\mathcal{O}_{4 F}^{(8)}$.

The indirect parity violation induces a mixing between an even-parity state and an oddparity state as well as a S-state and a P-state in a quarkonium. As the parity violating term is written by $\delta V^{\mu \nu}(r)$ in the potential, the effective action in Eq. (A.21) includes indirect parity violation by rewriting $V(r) g^{\mu \nu} \rightarrow V(r) g^{\mu \nu}+\delta V^{\mu \nu}(r)$. Here $\delta V^{\mu \nu}(r)$ is a matrix in a basis of $\mathrm{S}$ - and P-states, which has off-diagonal elements of hadron state labeled by $n$ (and $\mu$ ). Now let us calculate the mixing between asymptotic states in the SUSY SM by using the basis of the SM QCD. Since the potential only depends on relative coordinate, the wave function can be expanded by $\Psi_{n}(\vec{r})$ in the SUSY SM as

$$
\phi_{X}^{\mu}(\vec{r})=\sum_{n} A_{n}^{\mu}(X) \Psi_{n}(\vec{r})
$$

where $\Psi_{n}(\vec{r})$ satisfies eignvalue equations,

$$
\left[H^{\mathrm{QCD}}(\vec{r})+\delta V(\vec{r})\right] \Psi_{n}(\vec{r})=E_{n}^{\text {full }} \Psi_{n}(\vec{r}),
$$

for $E_{n}^{\text {full }} \neq E_{n}$. Note that $n$ is the label of the hadron, which contains an information of spin ( $\mu=0$ : singlet, $\mu=i$ : triplet). This $\Psi_{n}(\vec{r})$ must be $\Psi_{n}(\vec{r}) \rightarrow \psi_{n}(\vec{r})$ as $\delta V \rightarrow 0$, so that it is given by

$$
\Psi_{n}(\vec{r})=\psi_{n}(\vec{r})+\sum_{k \neq n} \frac{V_{n k}}{E_{n}-E_{k}} \psi_{k}(\vec{r})
$$


up to the first order of perturbation. Note that $\Psi_{n}(\vec{r})$ must satisfy

$$
\int d^{3} r \Psi_{n}^{\dagger}(\vec{r}) \Psi_{m}(\vec{r})=\delta_{m n}
$$

for the zeroth order of perturbation. $V_{n k}$ is defined by

$$
V_{n k} \equiv \int d^{3} s \psi_{k}^{\dagger}(\vec{s})[\delta V(\vec{s})] \psi_{n}(\vec{s})
$$

The classical complete set $\Psi_{n}(\vec{r})$ should be written by the QCD complete set $\psi_{n}(\vec{r})$, while a hadron creation operator is given by $A_{n}^{\mu \dagger}(X)$, so that $A_{n}^{\mu}(X)$ corresponds to $a_{n}^{\mu}(X) . \Psi_{n}(\vec{r})$ and $\psi_{n}(\vec{r})$ are different complete bases as

$$
\phi_{X}^{\mu}(\vec{r})=\sum_{n} a_{n}^{\mu}(X) \psi_{n}(\vec{r})=\sum_{n} A_{n}^{\mu}(X) \Psi_{n}(\vec{r}) .
$$

Thus, by use of orthogonalization of $\Psi_{n}(\vec{r})$, we obtain

$$
A_{n}^{\mu \dagger}(X)=a_{n}^{\mu \dagger}(X)+\sum_{k \neq n} \frac{\left(V_{n k} a_{k}^{\dagger}(X)\right)^{\mu}}{E_{n}-E_{k}} .
$$

Let us consider a charmonium system. Equation (4.7) means an observed $\eta_{c}$ is almost represented by a mixing state of $\eta_{c}$ and $\chi_{c 0}$ as

$$
\left|\eta_{c}\right\rangle_{\mathrm{obs} .}=\left|\eta_{c}\right\rangle+\frac{V_{\eta_{c}, \chi_{c 0}}}{E_{\eta_{c}}-E_{\chi_{c 0}}}\left|\chi_{c 0}\right\rangle
$$

$\chi_{c 0}$ is $0^{++}$with mass of $3415 \mathrm{MeV}$, and a decay of $\chi_{c 0}$ to $\pi \pi$ is possible (see, Eq.(5.2) ) when $\pi-\pi$ system has angular momentum, $L=1$. We estimate parity violating potential induced from the SUSY SM. As for $\mathcal{O}_{4 F}^{(1)}$ in Fig. 2, its coefficient only depends on $m_{\tilde{c}_{L}}$ and $m_{\tilde{c}_{R}}$, since the bound state is charmonium. The parity violating terms in $\mathcal{O}_{4 F}^{(1)}$ are given by

$$
\mathcal{O}_{4 F}^{(1)} \supset \frac{12 g_{s}^{4}}{192 \pi^{2}} \frac{1}{2}\left(-C_{L L}^{(\tilde{c}, \tilde{c})}+C_{R R}^{(\tilde{c}, \tilde{c})}\right) \delta^{4}(x-y)\left[\bar{c}(x) \gamma^{\mu} c(x)\right]\left[\bar{c}(y) \gamma_{\mu} \gamma^{5} c(y)\right]
$$

where we use spin relation, $\delta_{\alpha \beta} \delta_{\gamma \lambda}=\frac{1}{2} \delta_{\alpha \lambda} \delta_{\gamma \beta}+\frac{1}{2} \sigma_{\alpha \lambda}^{a} \sigma_{\gamma \beta}^{a}$, and $\sigma^{a} \sigma^{b}=\delta^{a b}+i \epsilon^{a b c} \sigma^{c}$. A color factor is rewritten as $\frac{1}{2} \delta_{i j} \delta_{k l}=\frac{1}{2 N_{C}} \delta_{i l} \delta_{k j}+T_{i l}^{A} T_{k j}^{A}$ for an exchange of spin. We must be careful for exchanges of spin and coordinate, where only spin-singlet changes its sign (Table1). After careful calculations, $\mathcal{O}_{4 F}^{(1)}$ is given by

$$
\begin{aligned}
\mathcal{O}_{4 F}^{(1)} & \rightarrow \frac{12 g_{s}^{4}}{192 \pi^{2}} \frac{1}{2}\left(-C_{L L}^{(\tilde{c}, \tilde{c})}+C_{R R}^{(\tilde{c}, \tilde{c})}\right)\left(\frac{i}{4 m_{c} N_{C}}\right) \\
& \times\left(\begin{array}{c}
\phi^{0} \\
\phi^{i}
\end{array}\right)_{x, y}^{\dagger}\left(\begin{array}{cc}
0 & 4 \mathcal{V}(r) \partial_{r}^{j} \\
4 \overleftarrow{\partial}{ }_{r}^{i} \mathcal{V}(r) & 4 i \epsilon^{i j k} \overleftarrow{\partial}_{r}^{k} \mathcal{V}(r)
\end{array}\right)\left(\begin{array}{c}
\phi^{0} \\
\phi^{j}
\end{array}\right)_{x, y},
\end{aligned}
$$




\begin{tabular}{|l|c|c|}
\hline & exchange of spin $(\varphi \leftrightarrow \chi)$ & exchange of coordinate $(x \leftrightarrow y)$ \\
\hline spin singlet $\phi^{0}(x, y)$ & asym. & sym. \\
spin triplet $\phi^{i}(x, y)$ & sym. & asym. \\
\hline
\end{tabular}

Table 1: exchanges of spin or coordinate

where $\mathcal{V} \equiv \delta^{4}(x-y)$ and $\phi^{i} \overleftarrow{\partial}_{r}^{i} \equiv-\partial_{r}^{i} \phi^{i}$. As for $\mathcal{O}_{4 F}^{(8)}$, we can use the calculation result of $\mathcal{O}_{4 F}^{(1)}$, since spin structure is the same. The different point is just color factor, and by using $T_{i j}^{A} T_{k l}^{A}=\frac{C_{F}}{2 N_{C}} \delta_{i l} \delta_{k j}-\frac{1}{N_{C}} T_{i l}^{A} T_{k j}^{A}$, we show color octet part is $C_{F}\left(=\left(N_{C}^{2}-1\right) /\left(2 N_{C}\right)\right)$ times larger than $\mathcal{O}_{4 F}^{(1)}$. Then, non-relativistic potential from $\mathcal{O}_{4 F}^{(1)}$ and $\mathcal{O}_{4 F}^{(8)}$ with parity violation is totally given by

$$
\begin{aligned}
& \delta V_{\mu \nu}^{4 F}(r) \\
& =\frac{12 g_{s}^{4}}{192 \pi^{2}} \frac{i}{8 m_{c} N_{C}}\left[\left(-C_{L L}^{(\tilde{c}, \tilde{c})}+C_{R R}^{(\tilde{c}, \tilde{c})}\right)+C_{F}\left(-D_{L L}^{(\tilde{c}, \tilde{c})}+D_{R R}^{(\tilde{c}, \tilde{c})}\right)\right]\left(\begin{array}{cc}
0 & 4 \mathcal{V}(r) \partial_{r}^{j} \\
4 \overleftarrow{\partial}{ }_{r}^{i} \mathcal{V}(r) & 4 i \epsilon^{i j k} \overleftarrow{\partial}{ }_{r}^{k} \mathcal{V}(r)
\end{array}\right) .
\end{aligned}
$$

For a non-relativistic potential from $\mathcal{O}_{q q G}$ in Eq.(B.14), we estimate leading part. Since $\mathcal{O}_{q q G}$ is not the contact interaction as $\mathcal{O}_{4 F}^{(1)}$, its parity violation effects should be added to the gluon potential. The bilocal operator after integrating out gluon is given by

$$
\mathcal{L} \sim \frac{g_{s}^{2}}{96 \pi^{2} C_{F}}\left[\bar{q}(x) T^{A} E_{L, R}^{0} P_{L, R} q(x)\right] V(r)\left[\bar{q}(y) T^{A} \gamma^{0} q(y)\right]
$$

where $E_{L, R}^{0}$ has eight terms in total, which are categorized as

$$
\begin{aligned}
& \text { (i) } \pm e_{1}\left(m_{\tilde{q}}\right) \frac{g_{s}^{2}}{96 \pi^{2} C_{F}}\left[\left(\bar{q}_{x} T^{A} \gamma_{\mu} \partial^{\mu} \partial^{0} q_{x}\right)+\left(\partial^{\mu} \partial^{0} \bar{q}_{x} T^{A} \gamma_{\mu} q_{x}\right)\right] V(r)\left[\bar{q}(y) T^{A} \gamma^{0} q(y)\right] \\
& \text { (ii) } \quad \pm e_{2}\left(m_{\tilde{q}}\right) \frac{g_{s}^{2}}{96 \pi^{2} C_{F}}\left[\left(\partial^{\mu} \bar{q}_{x} T^{A} \gamma_{\mu} \partial^{0} q_{x}\right)+\left(\partial^{0} \bar{q}_{x} T^{A} \gamma_{\mu} \partial^{\mu} q_{x}\right)\right] V(r)\left[\bar{q}(y) T^{A} \gamma^{0} q(y)\right] \\
&\left(\text { iii) } \pm \frac{g_{s}^{2}}{96 \pi^{2} C_{F}}\left[e_{3}\left(m_{\tilde{q}}\right)\left\{\left(\bar{q}_{x} T^{A} \gamma^{0} \partial^{2} q_{x}\right)+\left(\partial^{2} \bar{q}_{x} T^{A} \gamma^{0} q_{x}\right)\right\}+e_{4}\left(m_{\tilde{q}}\right)\left(\partial^{\mu} \bar{q}_{x} T^{A} \gamma^{0} \partial_{\mu} q_{x}\right)\right]\right. \\
& \quad \times V(r)\left[\bar{q}(y) T^{A} \gamma^{0} q(y)\right], \\
& \text { (iv) } \pm\left(-e_{5}\left(m_{\tilde{q}}\right)\right) \frac{g_{s}^{2}}{96 \pi^{2} C_{F}} i \epsilon^{\alpha \beta 0 \nu}\left[\partial_{\beta} \bar{q}_{x} T^{A} \gamma_{\nu} \partial_{\alpha} q_{x}\right] V(r)\left[\bar{q}(y) T^{A} \gamma^{0} q(y)\right] .
\end{aligned}
$$

Here, sign $+(-)$ means that quark chirality is $\mathrm{R}(\mathrm{L})$. In the non-relativistic limit, (i) and (ii) vanish, since components of $\mu=0$ and $\mu=i$ are cancelled with each other. For this calculation, we have used a NRQCD result, $\partial^{0} q \sim \mathcal{O}\left(\left(m_{c}^{2} v\right)^{3 / 2}\right)\left(v: c\right.$-quark velocity, $m_{c}: c$ quark mass). Actually, (iii) induces the leading effects for the potential. By taking leading 
order of $v$, a power counting shows

$$
\begin{aligned}
\delta V_{\mu \nu}^{q q G}(r) & =\left[\left(e_{4}\left(m_{\tilde{q}_{R}}\right)-e_{4}\left(m_{\tilde{q}_{L}}\right)\right)-2\left(e_{3}\left(m_{\tilde{q}_{R}}\right)-e_{3}\left(m_{\tilde{q}_{L}}\right)\right)\right] \\
& \times \frac{g_{s}^{2}}{96 \pi^{2}}\left(\frac{-i m_{q}}{8 N_{C}}\right)\left(\begin{array}{cc}
0 & V(r) \partial_{r}^{j}+\overleftarrow{\partial} \overleftarrow{r}^{j} V(r) \\
V(r) \partial_{r}^{i}+\overleftarrow{\partial}{ }_{r}^{i} V(r) & i \epsilon^{i j k}\left[V(r) \partial_{r}^{k}+\overleftarrow{\partial}{ }_{r}^{k} V(r)\right]
\end{array}\right),
\end{aligned}
$$

where we use color factor $\left(C_{F} /\left(2 N_{C}\right)\right)$ from Fierz transformation. As for (iv), $\alpha, \beta$ must be space-index, so that the second derivative of space-index appears, which corresponds to D-state (or higher angular momentum states), so that it does not contribute the mixing between Sand P-states. The (iv) does not contribute the mixing between S- and P-states, too. Thus, the leading order of parity violating potential, which triggers the mixing between $\mathrm{S}$ - and $\mathrm{P}$-states, is given by

$$
\delta V_{\mu \nu}^{\mathrm{SUSY}}(r)=\delta V_{\mu \nu}^{4 F}(r)+\delta V_{\mu \nu}^{q q G}(r)
$$

Then, we can calculate $V_{\eta_{c}, \chi_{c 0}}$ in a charmonium, and a formula of decay width is given by

$$
\Gamma\left(\eta_{c} \rightarrow \pi \pi\right) \sim\left|\frac{V_{\eta_{c}, \chi_{c 0}}}{E_{\eta_{c}}-E_{\chi_{c 0}}}\right|^{2} \Gamma\left(\chi_{c 0} \rightarrow \pi \pi\right) .
$$

A wave function of charmonium is given by $\psi(\vec{r})=R_{n}(r) Y_{l m}(\theta, \phi)$, where $R_{n}(r)$ satisfies the Schrödinger equation (2.3) with Coulomb plus linear potential (Cornell potential),

$$
V(r)=-\frac{\kappa}{r}+\frac{r}{a^{2}}
$$

We take $\kappa=0.52$ and $a=2.34 \mathrm{GeV}^{-1}$ for charmonium system [13]. Through the Schrödinger equation with this potential, we can obtain charmonium wave function numerically.

\section{Bounds for left-right non-degeneracy of squark masses}

We are in a stage to investigate bounds for left-right non-degeneracy of squark masses. At first, we investigate bounds for $\tilde{c}$ by use of calculation tools in this paper. Next, we estimate bounds for $\tilde{u}$ and $\tilde{d}$ by use of a similar technique in Ref.[14]. And finally, we comment on bounds for other sfermions.

\subsection{Bound for $\tilde{c}$}

Let us investigate the left-right non-degeneracy bound for the masses of $\tilde{c}_{L}$ and $\tilde{c}_{R}$ by use of the calculation method shown above. For a charmonium, we focus on $\eta_{c}$, whose decay has upper bounds of $P$ and $C P$ violations as [15]

$$
\operatorname{Br}\left(\eta_{c} \rightarrow \pi^{+} \pi^{-}\right)<6.0 \times 10^{-4}, \quad \operatorname{Br}\left(\eta_{c} \rightarrow \pi^{0} \pi^{0}\right)<4.0 \times 10^{-4}
$$


Note again that $\eta_{c}$ can not decay to $\pi \pi$ until it picks up parity violation. On the other hand, a branching ratio of $\chi_{c 0} \rightarrow \pi \pi$ is

$$
\operatorname{Br}\left(\chi_{c 0} \rightarrow \pi \pi\right)=(8.4 \pm 0.4) \times 10^{-3}
$$

A branching ratio of $\eta_{c} \rightarrow \pi \pi$ in a direct parity violation from Eq.(4.19) is given by

$$
\operatorname{Br}\left(\eta_{c} \rightarrow \pi \pi\right)_{\text {dir. }}=\left|A_{u c}+A_{d c}+B_{c u}+B_{c d}\right|^{2} \frac{\left|F^{s}\left(m_{\eta_{c}}\right)\right|^{2}\left|\psi_{\eta_{c}}(0)\right|^{2}}{16 m_{\eta_{c}}^{2} \Gamma_{\eta_{c}}},
$$

where $\Gamma_{\eta_{c}}$ is the total decay width of $\eta_{c}$. Here we take a scalar form factor of pion $F^{s}$ by an input parameter as $F^{s}\left(m_{\eta_{c}}^{2}\right)=1,0.1,0.001$, since its theoretical estimation is difficult above $1 \mathrm{GeV}$. On the other hand, the indirect parity violation in $\eta_{c} \rightarrow \pi \pi$ suggests

$$
\operatorname{Br}\left(\eta_{c} \rightarrow \pi \pi\right)_{\text {indir. }} \sim\left|\frac{V_{\eta_{c}, \chi_{c 0}}^{\mathrm{SUSY}}+V_{\eta_{c}, \chi_{c} 0}^{\mathrm{EW}}}{E_{\eta_{c}}-E_{\chi_{c 0}}}\right|^{2} \operatorname{Br}\left(\chi_{c 0} \rightarrow \pi \pi\right),
$$

where $V_{\eta_{c}, \chi_{c 0}}^{\mathrm{EW}}$ is the SM background induced from a $Z$-boson exchange. It gives an additional effect $\left.\mathcal{V}(r) \equiv(\alpha / r) \exp \left(-m_{Z} r\right)\right)$ in Eq.(4.11), which is shown as

$$
\delta V_{\mu \nu}^{\mathrm{EW}}(r)=\frac{g^{2}}{\cos ^{2} \theta_{W}}\left(\frac{1}{2}-\frac{2}{3} \sin ^{2} \theta_{W}\right)^{2} \frac{i C_{F}}{8 m_{c} N}\left(\begin{array}{cc}
0 & 4 \mathcal{V}(r) \partial_{r}^{j} \\
4 \overleftarrow{\partial}{ }_{r}^{i} \mathcal{V}(r) & 4 i \epsilon^{i j k} \overleftarrow{\partial}{ }_{r}^{k} \mathcal{V}(r)
\end{array}\right)
$$

in a basis of (S-state, P-state) with $N=2$ and $C_{F}=3 / 2$. Then, we can evaluate $V_{\eta_{c}, \chi_{c 0}}^{\mathrm{EW}}$ with Eq.(4.5), and the branching ratio is given by $\operatorname{Br}\left(\eta_{c} \rightarrow \pi \pi\right)_{\mathrm{SM}} \sim\left|\frac{V_{\eta_{c}, \chi_{c 0}}^{\mathrm{EW}}}{E_{\eta_{c}}-E_{\chi_{c 0} 0}}\right|^{2} \operatorname{Br}\left(\chi_{c 0} \rightarrow \pi \pi\right) \simeq$ $7.0 \times 10^{-22}$.

In Figs. 3 and 4, the branching ratios of $\eta_{c} \rightarrow \pi \pi$ from direct and indirect parity violation effects are plotted, respectively, where horizontal axis is a magnitude of $\left(m_{\tilde{c}_{L}}^{2}-m_{\tilde{c}_{R}}^{2}\right) / m_{\tilde{g}}^{2}$. Note that the branching ratio from indirect parity violation is larger than that from direct parity violation. Unfortunately, we can show that the SUSY parity violating effect is smaller than the experimental bound of Eq. (5.1) in the parameter region, and it is difficult to obtain the non-degeneracy bound between $m_{\tilde{c}_{L}}$ and $m_{\tilde{c}_{R}}$. Figures 5 and 6 show a case that $\tilde{g}$ and $\tilde{c}_{R}$ are degenerate around $650 \mathrm{GeV}$ in mass. The magnitude of the horizontal axis is varied from $\left(m_{\tilde{c}_{L}}^{2}-m_{\tilde{c}_{R}}^{2}\right) / m_{\tilde{g}}^{2}=4.5$, which is taken to be consistent with LHC data. Notice that the branching ratio becomes larger than that in Figs. 3 and 4, however, the experimental bound is also much higher, and we can not obtain the bounds.

\subsection{Bounds for $\tilde{u}$ and $\tilde{d}$}

The left-right non-degeneracy bounds for $\tilde{u}$ and $\tilde{d}$ was studied by use of nuclear parity violation in Ref.[14. Where they compared coefficients of (quark level) meson-nucleon couplings in the 


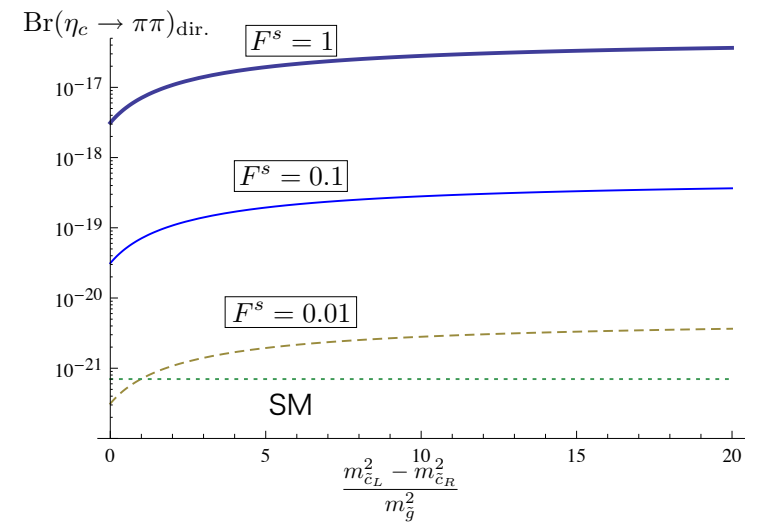

Figure 3: Branching ratios of $\eta \rightarrow \pi \pi$ from direct parity violation with $m_{\tilde{g}}=1000 \mathrm{GeV}, m_{\tilde{u}_{R}}=$ $1600 \mathrm{GeV}, m_{\tilde{u}_{L}}=2000 \mathrm{GeV}, m_{\tilde{d}_{R}}=1700 \mathrm{GeV}$, $m_{\tilde{d}_{L}}=2100 \mathrm{GeV}$, and $m_{\tilde{c}_{R}}=1800 \mathrm{GeV}$.

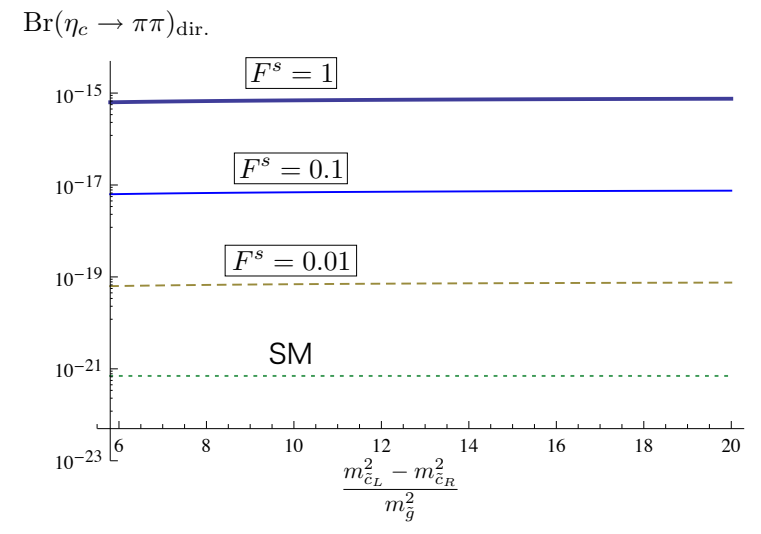

Figure 5: Branching ratios of $\eta \rightarrow \pi \pi$ from indirect parity violation with $m_{\tilde{g}}=850 \mathrm{GeV}, m_{\tilde{u}_{R}}=$ $860 \mathrm{GeV}, m_{\tilde{u}_{L}}=2000 \mathrm{GeV}, m_{\tilde{d}_{R}}=870 \mathrm{GeV}$, $m_{\tilde{d}_{L}}=2100 \mathrm{GeV}$, and $m_{\tilde{c}_{R}}=880 \mathrm{GeV}$.

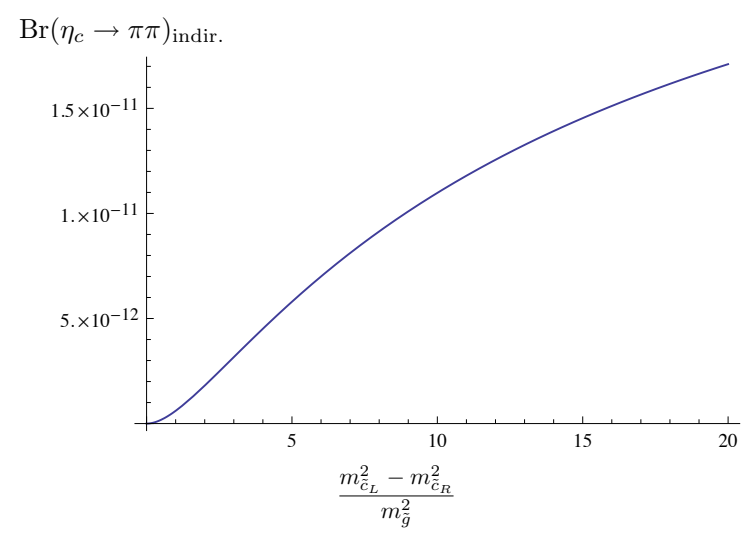

Figure 4: Branching ratios of $\eta \rightarrow \pi \pi$ from indirect parity violation with $m_{\tilde{g}}=1000 \mathrm{GeV}$, and $m_{\tilde{c}_{R}}=$ $1800 \mathrm{GeV}$.

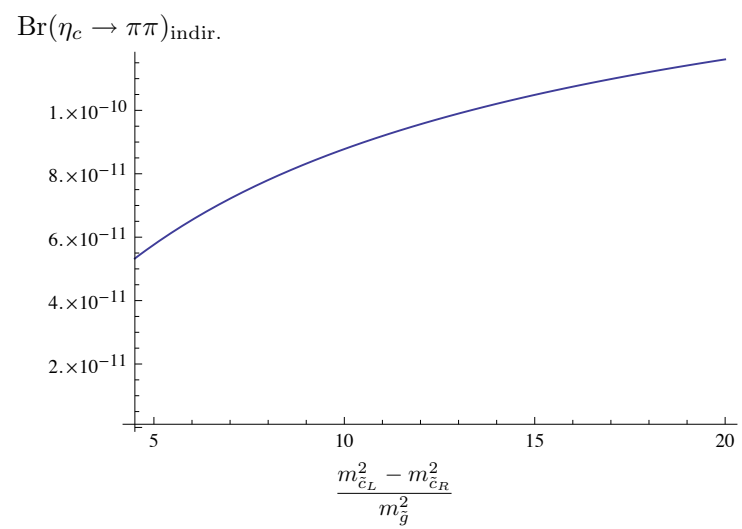

Figure 6: Branching ratios of $\eta \rightarrow \pi \pi$ from indirect parity violation with $m_{\tilde{g}}=850 \mathrm{GeV}$, and $m_{\tilde{c}_{R}}=$ $880 \mathrm{GeV}$. 
SM with those in the SUSY. However, studied parameter region was $m_{\tilde{q}}^{2} \ll m_{\tilde{g}}^{2}<\mathcal{O}\left(G_{F}^{-1}\right)$, which is already experimentally excluded, so that we investigate the left-right non-degeneracy bound in a wider parameter region, besides, without approximations used in Ref.[14].

We use $\pi, \omega, \rho$ and nucleon couplings for the meson-nucleon coupling. The notation of our dimension six operators corresponds to

$$
\frac{G_{2}\left(m_{\tilde{q}}, m_{\tilde{q}^{\prime}}\right)}{3 m_{\tilde{g}}^{2}}=f_{1}\left(m_{\tilde{q}}, m_{\tilde{q}^{\prime}}\right), \frac{G_{1}\left(m_{\tilde{q}}, m_{\tilde{q}^{\prime}}\right)}{3 m_{\tilde{g}}^{2}}=f_{2}\left(m_{\tilde{q}}, m_{\tilde{q}^{\prime}}\right),
$$

in Ref. [14], where we neglect flavor mixings and squark left-right mixings ( $A$-terms). On the other hand, coefficient of $q-q-G$ vertex is written by

$$
\frac{C\left(m_{\tilde{q}}^{2} / m_{\tilde{g}}^{2}\right)}{m_{\tilde{q}}^{2}}=\frac{43 m_{\tilde{g}}^{6}-144 m_{\tilde{g}}^{4} m_{\tilde{q}}^{2}+153 m_{\tilde{g}}^{2} m_{\tilde{q}}^{4}-6\left(2 m_{\tilde{g}}^{6}-9 m_{\tilde{g}}^{2} m_{\tilde{q}}^{4}+6 m_{\tilde{q}}^{6}\right) \log \left(\frac{m_{\tilde{g}}^{2}}{m_{\tilde{q}}^{2}}\right)-52 m_{\tilde{q}}^{6}}{54\left(m_{\tilde{g}}^{2}-m_{\tilde{q}}^{2}\right)^{4}}
$$

in a massless approximation of $u$ - and $d$-quarks. By using above equations, we calculate bounds from the SM as

$$
\begin{gathered}
\text { (i) }\left|C^{p}(\pi)+C_{-}^{b}(\pi)\right|<\left|C^{\mathrm{SEW}}(\pi)\right|, \\
\text { (ii) }\left|C^{p}(\omega)+C_{+}^{b}(\omega)\right|<\left|C^{\mathrm{SEW}}(\omega)\right|, \\
\text { (iii) }\left|C^{p}(\rho)+C_{+}^{b}(\rho)\right|<\left|C^{\mathrm{SEW}}(\rho)\right|,
\end{gathered}
$$

which are shown in Figs. 7, 8, and 9, $C(\pi), C(\omega)$, and $C(\rho)$ are parity violating effects (coupling) from $\pi-, \omega$, and $\rho$-nucleon interactions, respectively. Indcies $p$ and $b$ stand for penguin and box diagram contributions, respectively. Index SEW means an effect from the $\mathrm{SM}$ electroweak interactions [14] as $\left|C^{\mathrm{SEW}}(\pi)\right|=8.5 \times 10^{-7},\left|C^{\mathrm{SEW}}(\omega)\right|=4.5 \times 10^{-6}$, and $\left|C^{\mathrm{SEW}}(\rho)\right|=6.2 \times 10^{-7}$. The factor $c\left(m_{\tilde{q}}\right)$ is defined by $c\left(m_{\tilde{q}}\right) \equiv C\left(m_{\tilde{q}}^{2} / m_{\tilde{g}}^{2}\right) / m_{\tilde{q}}^{2}$, and then

$$
\begin{aligned}
& C^{p}(\pi)=\frac{4}{3} \frac{\alpha_{s}^{2}}{12} \rho\left[c\left(m_{\tilde{u}_{R}}\right)-c\left(m_{\tilde{u}_{L}}\right)-c\left(m_{\tilde{d}_{R}}\right)+c\left(m_{\tilde{d}_{L}}\right)\right], \\
& C^{p}(\omega)=\frac{1}{3} \frac{\alpha_{s}^{2}}{24} \rho\left[c\left(m_{\tilde{u}_{R}}\right)-c\left(m_{\tilde{u}_{L}}\right)+c\left(m_{\tilde{d}_{R}}\right)-c\left(m_{\tilde{d}_{L}}\right)\right], \\
& C^{p}(\rho)=\frac{2}{3} \frac{\alpha_{s}^{2}}{24} \rho\left[c\left(m_{\tilde{u}_{R}}\right)-c\left(m_{\tilde{u}_{L}}\right)+c\left(m_{\tilde{d}_{R}}\right)-c\left(m_{\tilde{d}_{L}}\right)\right], \\
& C_{-}^{b}(\pi)=-\frac{\alpha_{s}^{2}}{27} \rho\left[f_{1}\left(m_{\tilde{u}_{L}}, m_{\tilde{d}_{R}}\right)-f_{1}\left(m_{\tilde{u}_{R}}, m_{\tilde{d}_{L}}\right)-f_{2}\left(m_{\tilde{u}_{L}}, m_{\tilde{d}_{R}}\right)+f_{2}\left(m_{\tilde{u}_{R}}, m_{\tilde{d}_{L}}\right)\right],
\end{aligned}
$$




$$
\begin{aligned}
C_{+}^{b}(\omega) & =-\frac{3 \alpha_{s}^{2}}{48}\left(\frac{2}{9}+\frac{8}{27}\right) \rho\left[2 f_{1}\left(m_{\tilde{u}_{L}}, m_{\tilde{d}_{L}}\right)-2 f_{1}\left(m_{\tilde{u}_{R}}, m_{\tilde{d}_{R}}\right)-f_{2}\left(m_{\tilde{u}_{L}}, m_{\tilde{d}_{L}}\right)+2 f_{2}\left(m_{\tilde{u}_{R}}, m_{\tilde{d}_{R}}\right)\right. \\
& -f_{1}\left(m_{\tilde{d}_{L}}, m_{\tilde{d}_{L}}\right)-f_{1}\left(m_{\tilde{d}_{R}}, m_{\tilde{d}_{R}}\right)-f_{1}\left(m_{\tilde{u}_{L}}, m_{\tilde{u}_{L}}\right)-f_{1}\left(m_{\tilde{u}_{R}}, m_{\tilde{u}_{R}}\right) \\
& \left.+f_{2}\left(m_{\tilde{d}_{L}}, m_{\tilde{d}_{L}}\right)+f_{2}\left(m_{\tilde{d}_{R}}, m_{\tilde{d}_{R}}\right)+f_{2}\left(m_{\tilde{u}_{L}}, m_{\tilde{u}_{L}}\right)+f_{2}\left(m_{\tilde{u}_{R}}, m_{\tilde{u}_{R}}\right)\right] \\
C_{+}^{b}(\rho) & =-\frac{\alpha_{s}^{2}}{48} \frac{32}{27} \rho\left[2 f_{1}\left(m_{\tilde{u}_{L}}, m_{\tilde{d}_{L}}\right)-2 f_{1}\left(m_{\tilde{u}_{R}}, m_{\tilde{d}_{R}}\right)-f_{2}\left(m_{\tilde{u}_{L}}, m_{\tilde{d}_{L}}\right)+2 f_{2}\left(m_{\tilde{u}_{R}}, m_{\tilde{d}_{R}}\right)\right. \\
& -f_{1}\left(m_{\tilde{d}_{L}}, m_{\tilde{d}_{L}}\right)-f_{1}\left(m_{\tilde{d}_{R}}, m_{\tilde{d}_{R}}\right)-f_{1}\left(m_{\tilde{u}_{L}}, m_{\tilde{u}_{L}}\right)-f_{1}\left(m_{\tilde{u}_{R}}, m_{\tilde{u}_{R}}\right) \\
& \left.+f_{2}\left(m_{\tilde{d}_{L}}, m_{\tilde{d}_{L}}\right)+f_{2}\left(m_{\tilde{d}_{R}}, m_{\tilde{d}_{R}}\right)+f_{2}\left(m_{\tilde{u}_{L}}, m_{\tilde{u}_{L}}\right)+f_{2}\left(m_{\tilde{u}_{R}}, m_{\tilde{u}_{R}}\right)\right]
\end{aligned}
$$

where we take $\rho \sim \sqrt{10}$.

In Figs. 7, 8, and 9, we take sample points which are not excluded by ATLAS experiment [3, 16]. Under $m_{\tilde{g}}=1000 \mathrm{GeV}, m_{\tilde{u}_{R}}=1600 \mathrm{GeV}, m_{\tilde{u}_{L}}=2000 \mathrm{GeV}$, and $m_{\tilde{d}_{R}}=1700 \mathrm{GeV}$, we change a value of $\left(m_{\tilde{d}_{L}}^{2}-m_{\tilde{d}_{R}}^{2}\right) / m_{\tilde{g}}^{2}$ from 1.2 for the consistent with the experimental data. Unfortunately, in this parameter space, $\tilde{u}$ and $\tilde{d}$ are too heavy to obtain bounds for degeneracies between $m_{\tilde{u}_{L}}$ and $m_{\tilde{u}_{R}}$, or, $m_{\tilde{d}_{L}}$ and $m_{\tilde{d}_{R}}$. On the other hand, when gluino and squarks degenerate within $30 \mathrm{GeV}, \pi-, \omega$-, and $\rho$-nucleon couplings are shown in 10, 11, and 12, respectively. The magnitude of $\left(m_{\tilde{d}_{L}}^{2}-m_{\tilde{d}_{R}}^{2}\right) / m_{\tilde{g}}^{2}$ is varied from 4.5 for the consistency with the LHC data. In this parameter space, $\tilde{u}$ and $\tilde{d}$ are again too heavy to obtain the bounds. The branching ratio is small because SUSY effects always have a loop factor, and it is the reason why there are the asymptotic values in Figs. $7 \sim 12$.

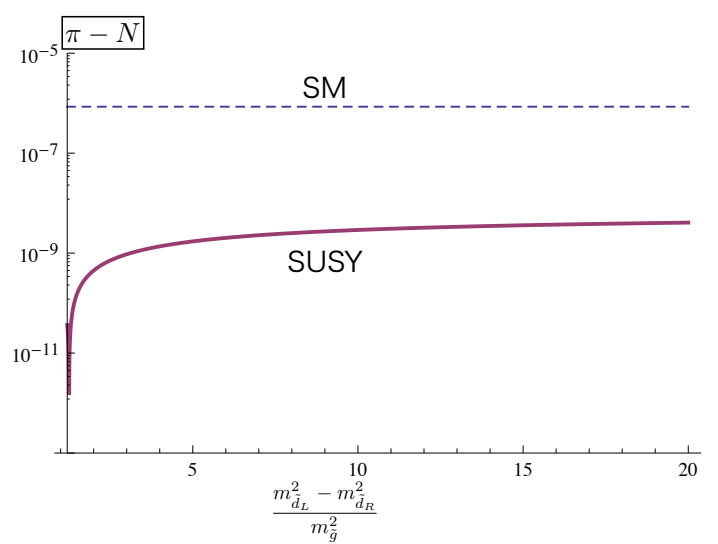

Figure 7: $\pi-N$ coupling $\left(\left|C^{p}(\pi)+C_{-}^{b}(\pi)\right|<\right.$ $\left.\left|C^{\mathrm{SEW}}(\pi)\right|\right)$ with $m_{\tilde{g}}=1000 \mathrm{GeV}, m_{\tilde{u}_{R}}=1600 \mathrm{GeV}$, $m_{\tilde{d}_{R}}=1700 \mathrm{GeV}$, and $m_{\tilde{u}_{L}}=2000 \mathrm{GeV}$. The magnitude closes in $7.1 \times 10^{-9}$ as $\left(m_{\tilde{d}_{L}}^{2}-m_{\tilde{d}_{R}}^{2}\right) / m_{\tilde{g}}^{2} \rightarrow \infty$.

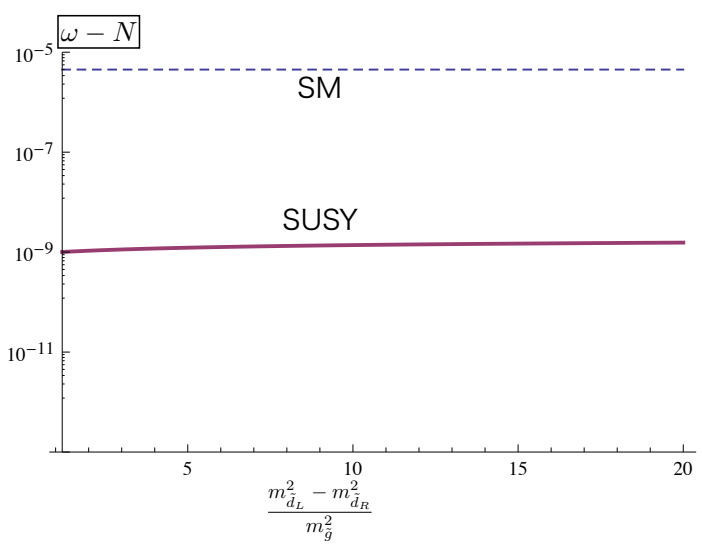

Figure 8: $\omega-N$ coupling $\left(\left|C^{p}(\omega)+C_{+}^{b}(\omega)\right|<\right.$ $\left.\left|C^{\mathrm{SEW}}(\omega)\right|\right)$ with $m_{\tilde{g}}=1000 \mathrm{GeV}, m_{\tilde{u}_{R}}=1600 \mathrm{GeV}$, $m_{\tilde{d}_{R}}=1700 \mathrm{GeV}$, and $m_{\tilde{u}_{L}}=2000 \mathrm{GeV}$. The magnitude closes in $2.1 \times 10^{-9}$ as $\left(m_{\tilde{d}_{L}}^{2}-m_{\tilde{d}_{R}}^{2}\right) / m_{\tilde{g}}^{2} \rightarrow \infty$. 


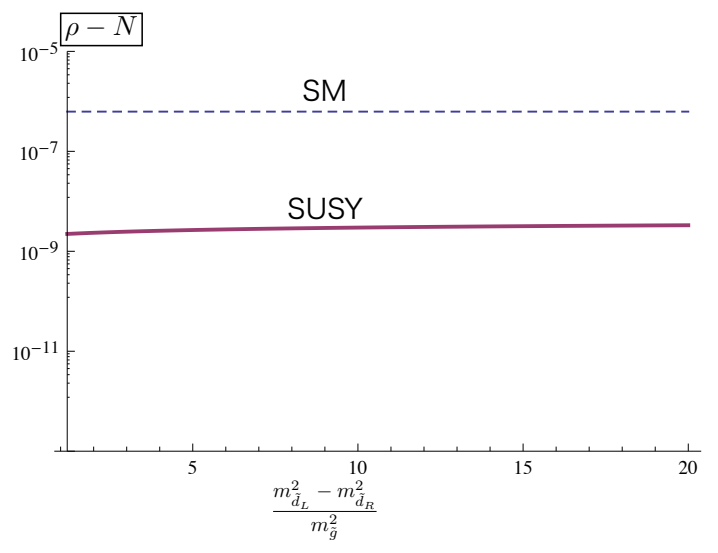

Figure 9: $\quad \rho-N$ coupling $\left(\left|C^{p}(\rho)+C_{+}^{b}(\rho)\right|<\right.$ $\left.\left|C^{\mathrm{SEW}}(\rho)\right|\right)$ with $m_{\tilde{g}}=1000 \mathrm{GeV}, m_{\tilde{u}_{R}}=1800 \mathrm{GeV}$, $m_{\tilde{d}_{R}}=1700 \mathrm{GeV}$, and $m_{\tilde{u}_{L}}=2000 \mathrm{GeV}$. The magnitude closes in $4.4 \times 10^{-9}$ as $\left(m_{\tilde{d}_{L}}^{2}-m_{\tilde{d}_{R}}^{2}\right) / m_{\tilde{g}}^{2} \rightarrow \infty$.

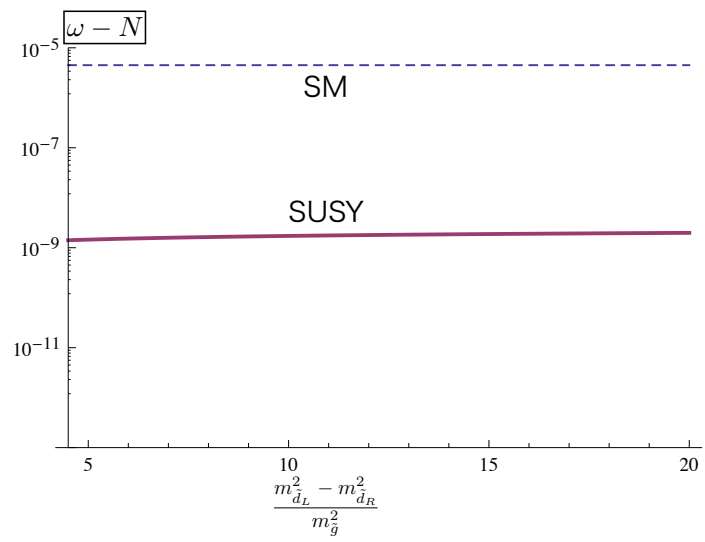

Figure 11: $\omega-N$ coupling $\left(\left|C^{p}(\omega)+C_{+}^{b}(\omega)\right|<\right.$ $\left.\left|C^{\mathrm{SEW}}(\omega)\right|\right)$ with $m_{\tilde{g}}=850 \mathrm{GeV}, m_{\tilde{u}_{R}}=860 \mathrm{GeV}$, $m_{\tilde{d}_{R}}=870 \mathrm{GeV}$, and $m_{\tilde{u}_{L}}=2000 \mathrm{GeV}$. The magnitude closes in $2.7 \times 10^{-9}$ as $\left(m_{\tilde{d}_{L}}^{2}-m_{\tilde{d}_{R}}^{2}\right) / m_{\tilde{g}}^{2} \rightarrow \infty$.

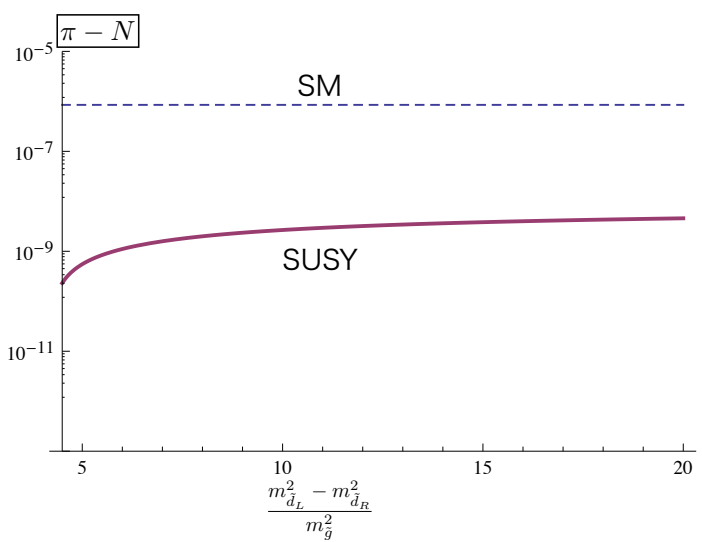

Figure 10: $\pi-N$ coupling $\left(\left|C^{p}(\pi)+C_{-}^{b}(\pi)\right|<\right.$ $\left.\left|C^{\mathrm{SEW}}(\pi)\right|\right)$ with $m_{\tilde{g}}=850 \mathrm{GeV}, m_{\tilde{u}_{R}}=860 \mathrm{GeV}$, $m_{\tilde{d}_{R}}=870 \mathrm{GeV}$, and $m_{\tilde{u}_{L}}=2000 \mathrm{GeV}$. The magnitude closes in $9.0 \times 10^{-9}$ as $\left(m_{\tilde{d}_{L}}^{2}-m_{\tilde{d}_{R}}^{2}\right) / m_{\tilde{g}}^{2} \rightarrow \infty$.

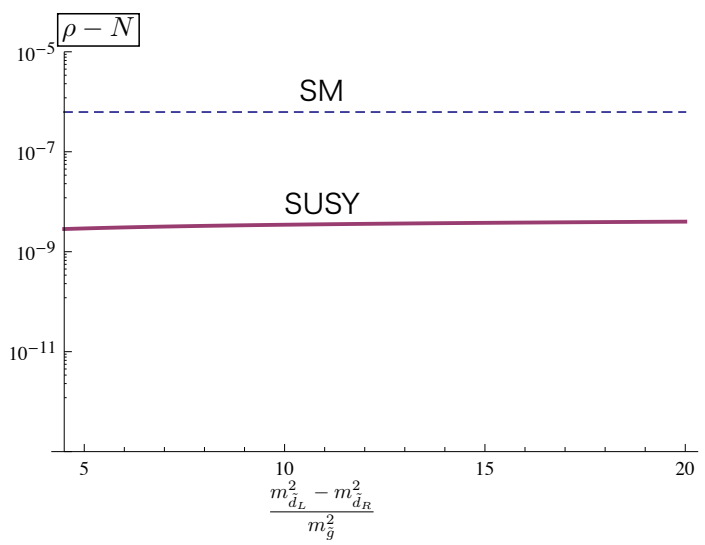

Figure 12: $\quad \rho-N$ coupling $\left(\left|C^{p}(\rho)+C_{+}^{b}(\rho)\right|<\right.$ $\left.\left|C^{\mathrm{SEW}}(\rho)\right|\right)$ with $m_{\tilde{g}}=850 \mathrm{GeV}, m_{\tilde{u}_{R}}=860 \mathrm{GeV}$, $m_{\tilde{d}_{R}}=870 \mathrm{GeV}$, and $m_{\tilde{u}_{L}}=2000 \mathrm{GeV}$. The magnitude closes in $5.6 \times 10^{-9}$ as $\left(m_{\tilde{d}_{L}}^{2}-m_{\tilde{d}_{R}}^{2}\right) / m_{\tilde{g}}^{2} \rightarrow \infty$. 


\subsection{Bounds for other sfermions}

Let us comment on the bounds for left-right non-degeneracies of other sfermions. As for $\tilde{b}$, a total decay width of each bound state of $b \bar{b}$-meson has not experimentally measured yet. If we can know the width, the $b \bar{b}$-meson system can be analyzed, and a bound for a nondegeneracy between $m_{\tilde{b}_{L}}$ and $m_{\tilde{b}_{R}}$ can be calculated just as the bound between $m_{\tilde{c}_{L}}$ and $m_{\tilde{c}_{R}}$ was calculated from the charmonium. We will calculate the bounds by just replacing $\eta_{c} \rightarrow \eta_{b}$ $\left(\eta_{b}: 0^{-+}\right)$and $\chi_{c 0} \rightarrow \chi_{b 0}\left(\chi_{b 0}: 0^{++}\right)$. We hope our method is useful to give a bound between $m_{\tilde{b}_{L}}$ and $m_{\tilde{b}_{R}}$ from a future experiments of $B$-physics.

As for $\tilde{s}$, it is difficult to estimate the bound from the same method in section 6.1. The reason is as follows. If we include a mixing between $d$ - and $s$-quarks through the Cabbibo angle, this effect is too small to induce the bound between $m_{\tilde{s}_{L}}$ and $m_{\tilde{s}_{R}}$ because Figs. 7 , 8, and 9 can not give bounds for $\tilde{u}, \tilde{d}$, too. On the other hand, if we take $s$-quark as a heavy quark and calculate a quarkonium in NRQCD as $c$-quark, we might have bounds of $\tilde{s}$ for leftright non-degeneracy from parity violating decay mode of $\eta(548)$. Here, $\eta(548)$ is $0^{-+}$which might have a mixing with $f_{0}(600)\left(0^{++}\right)$, if parity violation exists. The decay mode of $f_{0}(600)$ is dominated by $2 \pi$. Thus, the parity violation induces $\eta(548) \rightarrow \pi \pi$, whose experimental bounds are given by

$$
\operatorname{Br}\left(\eta \rightarrow \pi^{+} \pi^{-}\right)<1.3 \times 10^{-5}, \quad \operatorname{Br}\left(\eta \rightarrow 2 \pi^{0}\right)<3.5 \times 10^{-4} .
$$

However, these state are not composed only by $s$-quarks but also $u$-, $d$-quarks, so that a valid estimation is difficult. Also we should remind that mass of $s$-quark is about ten times smaller than that of $c$-quark which is too light to be treated in the NRQCD.

Finally, we comment on sleptons. Lepton flavor violation (LFV) experiments require stringent bounds of non-degeneracy among slepton flavors (generations). However, the LFV is suppressed when slepton masses are heavy enough even if their left- and right-handed slepton masses are not degenerate. That is, the left-right degeneracy is not required when sleptons are heavy enough. This situation is the same for squark sector as above (and also shown in $K^{0}-\bar{K}^{0}$ system, where left-right degeneracy is not required with enough heavy squarks).

\section{Summary}

The SUSY SM undergoes parity violation in QCD through chiral quark-squark-gluino interactions with non-degenerate masses between left-handed and right-handed squarks. Since experiments have not shown any parity violation in QCD yet, a bound for the mass degeneracy between left-handed and right-handed squarks should exist. We have tried to obtain this bound for each squark. First of all, we investigated the non-degeneracy bound between $m_{\tilde{c}_{L}}$ and $m_{\tilde{c}_{R}}$ from experimental data of charmonium decay by use of NRQCD. Second of all, we 
estimated the non-degeneracy bounds for $\tilde{u}$ and $\tilde{d}$ from nucleon-meson scattering data, and commented on other squarks. Unfortunately, our results are below current experimental data, and can not obtain the left-right degeneracy bounds for squark masses. We hope our method is useful for obtaining bounds from future experimental data.

\section{Acknowledegements}

We thank K. Hanagaki, K. Hikasa, T. Sato, M. Wakamatsu, K. Hagiwara, C.S. Lim, Y. Kuno, T. Yamanaka, and S. Kishimoto, for useful and helpful discussions. We are also grateful to W. Naylor and C. Kevin for careful reading of the manuscript. This work is partially supported by Scientific Grant by Ministry of Education and Science, Nos. 20540272, 22011005, 20244028, and 21244036 .

\section{A Two-body state effective action}

Here we derive the effective action of heavy $q \bar{q}$-system in NRQCD, Eq.(2.1). At leading order in perturbation theory, we can write an effective QCD Lagrangian as

$$
S=\int_{x}[\bar{q}(i \not \partial-m) q]+(-i) \int_{x} \int_{y} j_{\mu}^{\dagger}(x) \mathcal{D}^{\mu \nu}(x-y) j_{\nu}(y),
$$

where $\mathcal{D}^{\mu \nu}(x-y)$ is gluon propagator. In non-relativistic limit, the gluon propagator induces a (gluon) potential as

$$
\begin{aligned}
\mathcal{D}^{\mu \nu}(x-y) & =\int \frac{d^{4} p}{(2 \pi)^{4}} \frac{-i g_{s}^{2} g^{\mu \nu}}{p_{0}^{2}-|\vec{p}|^{2}} e^{-i p \cdot(x-y)} \\
& \simeq \delta\left(x^{0}-y^{0}\right) \frac{i g_{s}^{2} g^{00}}{4 \pi|\vec{x}-\vec{y}|} \equiv i \delta\left(x^{0}-y^{0}\right) \frac{V(r)}{C_{F}}
\end{aligned}
$$

where $r=|\vec{x}-\vec{y}|$ and $V(r) \equiv C_{F} g_{s}^{2} /(4 \pi r)$. This is the "Coulomb" potential when energy level of $q \bar{q}$-system is low (for example, S-state in $c \bar{c}$-system, $\eta_{c}$ ). For high energy levels (for example, $\mathrm{P}$ state in $c \bar{c}$-system, $\left.\chi_{c}\right)$ the potential of the heavy $q \bar{q}$-system $V(r)$ should be well approximated by phenomenological potential such a "Coulomb" plus linear as Eq.(4.20). This is because, at longer distance, higher-order perturbation such as gluon self interaction gets more important. In fact, Refs. [17, 18, 19] show that the perturbatively calculated QCD potential agrees with lattice calculations or phenomenologically suggested potential. When $q$ is a heavy quark, it is expanded by its mass as

$$
q(x)=\left(\begin{array}{c}
\varphi e^{-i m t}+i \frac{\vec{\nabla} \cdot \vec{\sigma}}{2 m} \chi e^{i m t} \\
\chi e^{i m t}-i \frac{\vec{\nabla} \cdot \vec{\sigma}}{2 m} \varphi e^{-i m t}
\end{array}\right)
$$


$\varphi$ and $\chi$ denote particle and anti-particle components, respectively, and this expansion is so-called Foldy-Wouthuysen-Tani transformation[20, 21]. Taking a color singlet part in the second term of Eq.(A.1) (color octet part is the next leading order [5]), we can obtain a NRQCD action,

$$
\begin{aligned}
& S_{\mathrm{NRQCD}}=\int_{x}\left[\varphi^{\dagger}\left(i \partial_{0}+\frac{\nabla^{2}}{2 m}\right) \varphi+\chi^{\dagger}\left(i \partial_{0}-\frac{\nabla^{2}}{2 m}\right) \chi\right] \\
& +\frac{1}{2 N_{C}} \int_{x} \int_{y} \delta\left(x^{0}-y^{0}\right)\left[\varphi^{\dagger}(x) \chi(y) V(r) \chi^{\dagger}(y) \varphi(x)+\varphi^{\dagger}(x) \sigma^{i} \chi(y) V(r) \chi^{\dagger}(y) \sigma_{i} \varphi(x)\right],
\end{aligned}
$$

where color factor comes from $T_{i j}^{A} T_{k l}^{A}=\frac{C_{F}}{2 N_{C}} \delta_{i l} \delta_{k j}-\frac{1}{N_{C}} T_{i l}^{A} T_{k j}^{A}$ through Fierz transformation. Hereafter, we note $N_{C}$ as a color number, which is, of cause, $N_{C}=3$. Next, by inserting the following identities,

$$
\begin{aligned}
& 1=\int \prod_{\mu, \nu} \mathcal{D} s^{\mu} \mathcal{D} \phi^{\nu \dagger} \exp i \int_{x} \int_{y} \phi_{\mu}^{\dagger}(x, y)\left(s^{\mu}(x, y)-\varphi^{\dagger}(x) \sigma^{\mu} \chi(y)\right), \\
& 1=\int \prod_{\mu, \nu} \mathcal{D} s^{\mu \dagger} \mathcal{D} \phi^{\nu} \exp i \int_{x} \int_{y} \phi_{\mu}(x, y)\left(s^{\mu \dagger}(x, y)-\chi^{\dagger}(x) \sigma^{\mu} \varphi(y)\right),
\end{aligned}
$$

into Eq.(A.4), the QCD action becomes

$$
\begin{aligned}
S_{\mathrm{NRQCD}} & =\int_{x} \int_{y}\left[\varphi^{\dagger}(x) K_{\varphi \varphi} \varphi(y)+\chi^{\dagger}(x) K_{\chi \chi} \chi(y)-\phi^{\mu \dagger}(x, y) \varphi^{\dagger}(x) \sigma_{\mu} \chi(y)-\chi^{\dagger}(x) \sigma_{\mu} \varphi(y) \phi^{\mu}(x, y)\right. \\
& \left.+\frac{1}{2 N_{C}} \delta\left(x^{0}-y^{0}\right) s^{\mu \dagger}(x, y) V(r) s_{\mu}(x, y)+\phi^{\mu \dagger}(x, y) s_{\mu}(x, y)+s_{\mu}^{\dagger}(x, y) \phi^{\mu}(x, y)\right], \quad \text { (A.7) }
\end{aligned}
$$

where the kinetic terms denote

$$
\begin{aligned}
& \varphi^{\dagger}(x) \delta^{4}(x-y)\left(i \partial_{0}+\frac{\nabla^{2}}{2 m}\right) \varphi(y) \equiv \varphi^{\dagger}(x) K_{\varphi \varphi} \varphi(y) \\
& \chi^{\dagger}(x) \delta^{4}(x-y)\left(i \partial_{0}-\frac{\nabla^{2}}{2 m}\right) \chi(y) \equiv \chi^{\dagger}(x) K_{\chi \chi} \chi(y)
\end{aligned}
$$

An effective action of the bilocal auxiliary field $\phi^{\mu}(x, y)$ will be obtained by integrating out $s^{\mu}, \varphi$, and $\chi$. A potential term is induced by integrating out $s^{\mu}$ as

$$
-\int_{x} \int_{y} \phi^{\mu \dagger}(x, y)\left[2 N_{C} \delta\left(x^{0}-y^{0}\right) V^{-1}(r) g_{\mu \nu}\right] \phi^{\nu}(x, y) .
$$

On the other hand, $\varphi$ - and $\chi$-integrations will derive a kinetic term of $\phi^{\mu}$ as shown below. We can rewrite the first four terms in Eq. A.7) as

$$
\int_{x} \int_{y}\left(\begin{array}{c}
\varphi(x) \\
\chi(x)
\end{array}\right)^{\dagger}\left(\begin{array}{cc}
K_{\varphi \varphi} & K_{\varphi \chi} \\
K_{\chi \varphi} & K_{\chi \chi}
\end{array}\right)\left(\begin{array}{l}
\varphi(y) \\
\chi(y)
\end{array}\right)
$$


where $K_{\varphi \chi}$ and $K_{\varphi \chi}$ are denoted as $K_{\varphi \chi}=-\phi^{\mu \dagger}(x, y) \sigma_{\mu}$ and $K_{\chi \varphi}=-\sigma_{\mu} \phi^{\mu}(x, y)$. Then,by integrating out $\varphi$ and $\chi$ in Eq.(A.7), we can obtain the term

$i \operatorname{Tr} \log \left(\begin{array}{cc}K_{\varphi \varphi} & K_{\varphi \chi} \\ K_{\chi \varphi} & K_{\chi \chi}\end{array}\right) \simeq i \operatorname{Tr} \log \left(\begin{array}{cc}K_{\varphi \varphi} & 0 \\ 0 & K_{\chi \chi}\end{array}\right)+i \sum_{n=1}^{\infty} \frac{(-1)^{n-1}}{n} \operatorname{Tr}\left(\begin{array}{cc}0 & K_{\varphi \varphi}^{-1} K_{\varphi \chi} \\ K_{\chi \chi}^{-1} K_{\chi \varphi} & 0\end{array}\right)^{n}$.

The Tr log is expanded in Eq. (A.12), where $n=1$ is vanished by a trace, and the leading term is coming from $n=2$. After taking traces of spinor, color, and coordinate indices, the leading term in Eq. (A.12) becomes

$$
\begin{aligned}
& \frac{-i}{2} \operatorname{Tr}\left(\begin{array}{cc}
K_{\varphi \varphi}^{-1} K_{\varphi \chi} K_{\chi \chi}^{-1} K_{\chi \varphi} & 0 \\
0 & K_{\chi \chi}^{-1} K_{\chi \varphi} K_{\varphi \varphi}^{-1} K_{\varphi \chi}
\end{array}\right) \\
& =-i N_{C} \int_{x} \int_{y} \int_{z} \int_{w} \operatorname{Tr}_{\text {spin }} K_{\varphi \varphi}^{-1}(x, y) K_{\varphi \chi}(y, z) K_{\chi \chi}^{-1}(z, w) K_{\chi \varphi}(w, x)
\end{aligned}
$$

where propagators are given by

$$
\begin{aligned}
& K_{\varphi \varphi}^{-1}(x, y)=\int \frac{d^{4} p}{(2 \pi)^{4}} \frac{1}{p^{0}-\frac{\vec{p}^{2}}{2 m}+i \epsilon} e^{-i p(x-y)} \delta^{\alpha \beta}, \\
& K_{\chi \chi}^{-1}(x, y)=-\int \frac{d^{4} q}{(2 \pi)^{4}} \frac{1}{q^{0}-\frac{\vec{q}^{2}}{2 m}+i \epsilon} e^{i q(x-y)} \delta^{\alpha \beta} .
\end{aligned}
$$

We use a center of mass coordinate $X^{\mu}$ and relative coordinate $(0, \vec{r})^{\mu}$ as $x^{\mu}=X^{\mu}+\frac{1}{2}(0, \vec{r})^{\mu}$ and $y^{\mu}=X^{\mu}-\frac{1}{2}(0, \vec{r})^{\mu}$. The relative coordinate does not have time-component, since $\phi^{\mu}(x, y)$ is a coincident bilocal field for $x$ and $y$. Then, $\phi^{\mu}(x, y)$ is represented by

$$
\phi^{\mu}(x, y) \equiv \phi_{X}^{\mu}(\vec{r})=\int_{k} \int_{l} \phi_{k}^{\mu}(\vec{l}) e^{-i k X} e^{-i l_{\mu}(0, \vec{r})^{\mu}}=\int_{k} \int_{l^{0}} \int_{\vec{l}} \phi_{k}^{\mu}(\vec{l}) e^{-i k X} e^{i \vec{l} \cdot \vec{r}},
$$

with their momentums as $p^{\mu}=\left(\frac{k^{0}}{2}+l^{0}, \frac{\vec{k}}{2}+\vec{l}\right)$ and $q^{\mu}=\left(\frac{k^{0}}{2}-l^{0}, \frac{\vec{k}}{2}-\vec{l}\right)$. In this frame, Eq. (A.13) is written as

$$
-2 N_{C} \int_{k} \int_{l} \int_{\vec{r}} \int_{\vec{s}} \frac{1}{\left[\frac{k^{0}}{2}+l^{0}-\frac{(\vec{k} / 2+\vec{l})^{2}}{2 m}+i \epsilon\right]\left[\frac{k^{0}}{2}-l^{0}-\frac{(\vec{k} / 2-\vec{l})^{2}}{2 m}+i \epsilon\right]} \phi_{k}^{\mu \dagger}(\vec{r}) \phi_{\mu k}(\vec{s}) e^{-i \vec{l} \cdot(\vec{r}+\vec{s})},
$$

and we obtain

$$
-2 i N_{C} \int_{k} \int_{\vec{l}} \int_{\vec{r}} \int_{\vec{s}} \frac{1}{k^{0}-\frac{\vec{k}^{2}}{4 m}-\frac{\overrightarrow{l^{2}}}{m}} \phi_{k}^{\mu \dagger}(\vec{r}) \phi_{\mu k}(\vec{s}) e^{-i \vec{l} \cdot(\vec{r}+\vec{s})}
$$


by integrating $l^{0}$. Then, the effective action of $\phi^{\mu}$ is given by

$$
S_{\text {eff }}=\int_{X} \int_{\vec{r}} \phi_{X}^{\mu \dagger}(\vec{r})\left[\frac{1}{V(r)}-\frac{1}{K_{X}(r)}\right] \phi_{\mu X}(\vec{r}),
$$

where $K_{X}(r) \equiv i \partial_{X}^{0}-\frac{\nabla_{X}^{2}}{4 m}-\frac{\nabla_{r}^{2}}{m}$. We omit overall factor $2 N_{C}$ by use of normalization of the field. Note that a Green function $\left\langle\phi_{X}^{\mu}(\vec{r}) \phi_{Y}^{\nu \dagger}(\vec{s})\right\rangle$ is given by

$$
\begin{aligned}
\left\langle\phi_{X}^{\mu}(\vec{r}) \phi_{Y}^{\nu \dagger}(\vec{s})\right\rangle & \equiv\left[V^{-1}-K^{-1}\right]_{\mu \nu}^{-1}(X, \vec{r} ; Y, \vec{s}) \\
& =V(r) g_{\mu \nu} \delta^{4}(X-Y) \delta^{3}(\vec{r}-\vec{s})+\left[V(K-V)^{-1} V\right]_{\mu \nu}(X, \vec{r} ; Y, \vec{s}) .
\end{aligned}
$$

In asymptotic states, $X \neq Y$, the first term vanishes. The second term is what we want, and $V$ is rotated out by field redefinition, then Eq. (A.19) becomes

$$
\left\langle\phi_{X}^{\mu}(\vec{r}) \phi_{Y}^{\nu \dagger}(\vec{s})\right\rangle=\left[(K-V)^{-1}\right]_{\mu \nu}(X, \vec{r} ; Y, \vec{s}) .
$$

This means that the effective action in Eq. A.18) can be rewritten as

$$
S_{\mathrm{eff}}=\int_{X} \int_{\vec{r}} \phi_{X}^{\mu \dagger}(\vec{r})\left[K_{X}(r)-V(r)\right] \phi_{\mu X}(\vec{r}) .
$$

This is the effective action of Eq.(2.1). We should notice that this form is correct when asymptotic states exist [22] and $\phi_{X}^{\mu}(\vec{r})$ is an on-shell state.

\section{B Dimension six operators from SUSY}

We calculate dimension six operators by intgrating out sparticles in the framework $R$-parity conservation. By integrating out SUSY particles, we can obtain higher order gauge invariant operators in terms of the SM fields. We calculate them up to $\mathcal{O}\left(\alpha_{s}^{2}\right)$ and neglect $\mathcal{O}\left(\alpha_{s} \alpha_{y}\right)$, where $\alpha_{s}\left(\alpha_{y}\right)$ is $g_{s}^{2} / 4 \pi\left(y^{2} / 4 \pi\right)$ with a QCD (Yukawa) coupling, $g_{s}(y)$. It is because Yukawa couplings, $y$ s, which we deal with are all small, and up to this order, sfermion left-right mixings are negligible. In the NRQCD, there are three types of dimension six operators, $\mathcal{O}_{4 F}^{(1)}, \mathcal{O}_{4 F}^{(8)}$, and $\mathcal{O}_{q q G}$, which can contribute the parity violation in QCD. Here $\mathcal{O}_{4 F}^{(1)}$ and $\mathcal{O}_{4 F}^{(8)}$ are color singlet and octet 4-Fermi operator, respectively. Other dimension six operators such as $q-q-G-G$ and $q-q-G-G-G$ vertexes are next leading order in the NRQCD, so we neglect them in the following discussions.

\footnotetext{
$\dagger$ We do not analyze $\tilde{t}$ in this paper.
} 
For $\mathcal{O}_{4 F}^{(1)}$ and $\mathcal{O}_{4 F}^{(8)}$, they are given by 23$]$

$$
\begin{aligned}
& \mathcal{O}_{4 F}^{(1)}=\frac{12 g_{s}^{4}}{192 \pi^{2}} \sum_{q, q^{\prime}}^{\text {flavor }}\left[C_{L L}\left(\bar{q} \gamma^{\mu} P_{L} q\right)\left(\bar{q}^{\prime} \gamma_{\mu} P_{L} q^{\prime}\right)+C_{R R}\left(\bar{q} \gamma^{\mu} P_{R} q\right)\left(\bar{q}^{\prime} \gamma_{\mu} P_{R} q^{\prime}\right)\right. \\
& \left.+C_{L R}\left(\bar{q} \gamma^{\mu} P_{L} q\right)\left(\bar{q}^{\prime} \gamma_{\mu} P_{R} q^{\prime}\right)+C_{R L}\left(\bar{q} \gamma^{\mu} P_{R} q\right)\left(\bar{q}^{\prime} \gamma_{\mu} P_{L} q^{\prime}\right)\right] \\
& \mathcal{O}_{4 F}^{(8)}=\frac{12 g_{s}^{4}}{192 \pi^{2}} \sum_{q, q^{\prime}}^{\text {flavor }}\left[D_{L L}\left(\bar{q} T^{a} \gamma^{\mu} P_{L} q\right)\left(\bar{q}^{\prime} T^{a} \gamma_{\mu} P_{L} q^{\prime}\right)+D_{R R}\left(\bar{q} T^{a} \gamma^{\mu} P_{R} q\right)\left(\bar{q}^{\prime} T^{a} \gamma_{\mu} P_{R} q^{\prime}\right)\right. \\
& \left.+D_{L R}\left(\bar{q} T^{a} \gamma^{\mu} P_{L} q\right)\left(\bar{q}^{\prime} T^{a} \gamma_{\mu} P_{R} q^{\prime}\right)+D_{R L}\left(\bar{q} T^{a} \gamma^{\mu} P_{R} q\right)\left(\bar{q}^{\prime} T^{a} \gamma_{\mu} P_{L} q^{\prime}\right)\right]
\end{aligned}
$$

where coefficients are

$$
\begin{gathered}
C_{L L}=\frac{2}{9}\left[f_{1}\left(m_{\tilde{q}_{L}}, m_{\tilde{q}_{L}^{\prime}}\right)+f_{2}\left(m_{\tilde{q}_{L}}, m_{\tilde{q}_{L}^{\prime}}\right)\right], \\
C_{R R}=\frac{2}{9}\left[f_{1}\left(m_{\tilde{q}_{R}}, m_{\tilde{q}_{R}^{\prime}}\right)+f_{2}\left(m_{\tilde{q}_{R}}, m_{\tilde{q}_{R}^{\prime}}\right)\right], \\
C_{L R}=-\frac{2}{9}\left[f_{1}\left(m_{\tilde{q}_{R}}, m_{\tilde{q}_{L}^{\prime}}\right)-f_{2}\left(m_{\tilde{q}_{L}}, m_{\tilde{q}_{R}^{\prime}}\right)\right], \\
C_{R L}=-\frac{2}{9}\left[f_{1}\left(m_{\tilde{q}_{L}}, m_{\tilde{q}_{R}^{\prime}}\right)+f_{2}\left(m_{\tilde{q}_{L}}, m_{\tilde{q}_{R}^{\prime}}\right)\right], \\
D_{L L}=-\frac{1}{3} f_{1}\left(m_{\tilde{q}_{L}}, m_{\tilde{q}_{L}^{\prime}}\right)-\frac{7}{6} f_{2}\left(m_{\tilde{q}_{L}}, m_{\tilde{q}_{L}^{\prime}}\right), \\
D_{R R}=-\frac{1}{3} f_{1}\left(m_{\tilde{q}_{R}}, m_{\tilde{q}_{R}^{\prime}}\right)-\frac{7}{6} f_{2}\left(m_{\tilde{q}_{R}}, m_{\tilde{q}_{R}^{\prime}}\right), \\
D_{L R}=-\frac{7}{6} f_{1}\left(m_{\tilde{q}_{L}}, m_{\tilde{q}_{R}^{\prime}}\right)-\frac{1}{3} f_{2}\left(m_{\tilde{q}_{L}}, m_{\tilde{q}_{R}^{\prime}}\right), \\
D_{R L}=-\frac{7}{6} f_{2}\left(m_{\tilde{q}_{R}}, m_{\tilde{q}_{L}^{\prime}}\right)-\frac{1}{3} f_{1}\left(m_{\tilde{q}_{R}}, m_{\tilde{q}_{L}^{\prime}}\right), \\
f_{1}\left(m_{\tilde{q}}, m_{\tilde{q}^{\prime}}\right)=\int_{0}^{1} d y \int_{0}^{1} d z \frac{z^{2}}{\left(m_{\tilde{g}}^{2}-m_{\tilde{q}}^{2}\right) y z+\left(m_{\tilde{q}}^{2}-m_{\tilde{q}^{\prime}}^{2}\right) z+m_{\tilde{q}^{\prime}}}, m_{\tilde{g}}^{2} y z^{2} \\
f_{2}\left(m_{\tilde{q}}, m_{\tilde{q}^{\prime}}\right)=\int_{0}^{1} d y \int_{0}^{1} d z \frac{\left[\left(m_{\tilde{g}}^{2}-m_{\tilde{q}}^{2}\right) y z+\left(m_{\tilde{q}}^{2}-m_{\tilde{q}^{\prime}}^{2}\right) z+m_{\tilde{q}^{\prime}}\right]^{2}}{} .
\end{gathered}
$$

On the other hand, $\mathcal{O}_{q q G}$ is given by

$$
\begin{aligned}
\mathcal{O}_{q q G} & =\frac{g_{s}^{3}}{96 \pi^{2}} \int \frac{d^{4} k_{1}}{(2 \pi)^{4}} \frac{d^{4} k_{2}}{(2 \pi)^{4}} \frac{d^{4} k_{3}}{(2 \pi)^{4}}(2 \pi)^{4} \delta^{4}\left(-k_{1}+k_{2}+k_{3}\right) \bar{q}\left(k_{2}\right) T^{a} E_{L, R}^{\mu} G_{\mu}^{a}\left(k_{3}\right) P_{L, R} q\left(k_{1}\right), \\
E_{L}^{\mu} & \equiv E^{\mu}\left(m_{\tilde{q}}=m_{\tilde{q}_{L}}\right) \\
& =\left\{e_{1}\left(m_{\tilde{q}_{L}}\right) \not k_{1}+e_{2}\left(m_{\tilde{q}_{L}}\right) \not k_{2}\right\} k_{1}^{\mu}+\left\{e_{1}\left(m_{\tilde{q}_{L}}\right) \not k_{2}+e_{2}\left(m_{\tilde{q}_{L}}\right) \not k_{1}\right\} k_{2}^{\mu} \\
& +\left\{e_{3}\left(m_{\tilde{q}_{L}}\right)\left(k_{1}^{2}+k_{2}^{2}\right)-e_{4}\left(m_{\tilde{q}_{L}}\right) k_{1} \cdot k_{2}\right\} \gamma^{\mu}-e_{5}\left(m_{\tilde{q}_{L}}\right) i \epsilon^{\alpha \beta \mu \nu} \gamma_{5} \gamma_{\nu} k_{1 \alpha} k_{2 \beta}, \\
E_{R}^{\mu} & =E^{\mu}\left(m_{\tilde{q}_{R}}\right)
\end{aligned}
$$




$$
\begin{aligned}
& e_{1}\left(m_{\tilde{q}}\right) \\
& =\frac{107 m_{\tilde{g}}^{6}-495 m_{\tilde{g}}^{4} m_{\tilde{q}}^{2}+477 m_{\tilde{g}}^{2} m_{\tilde{q}}^{4}-89 m_{\tilde{q}}^{6}-6\left(m_{\tilde{g}}^{6}+3 m_{\tilde{g}}^{4} m_{\tilde{q}}^{2}-54 m_{\tilde{g}}^{2} m_{\tilde{q}}^{4}+18 m_{\tilde{q}}^{6}\right) \log \left(m_{\tilde{g}}^{2} / m_{\tilde{q}}^{2}\right)}{18\left(m_{\tilde{g}}^{2}-m_{\tilde{q}}^{2}\right)^{4}} \\
& e_{2}\left(m_{\tilde{q}}\right) \\
& =\frac{-203 m_{\tilde{g}}^{6}+351 m_{\tilde{g}}^{4} m_{\tilde{q}}^{2}-189 m_{\tilde{g}}^{2} m_{\tilde{q}}^{4}+41 m_{\tilde{q}}^{6}+6\left(m_{\tilde{g}}^{6}+51 m_{\tilde{g}}^{4} m_{\tilde{q}}^{2}-54 m_{\tilde{g}}^{2} m_{\tilde{q}}^{4}+18 m_{\tilde{q}}^{6}\right) \log \left(m_{\tilde{g}}^{2} / m_{\tilde{q}}^{2}\right)}{18\left(m_{\tilde{g}}^{2}-m_{\tilde{q}}^{2}\right)^{4}} \\
& e_{3}\left(m_{\tilde{q}}\right)=e_{2}\left(m_{\tilde{q}}\right), \\
& e_{4}\left(m_{\tilde{q}}\right) \\
& =\frac{-155 m_{\tilde{g}}^{6}+423 m_{\tilde{g}}^{4} m_{\tilde{q}}^{2}-333 m_{\tilde{g}}^{2} m_{\tilde{q}}^{4}+65 m_{\tilde{q}}^{6}+6\left(m_{\tilde{g}}^{6}+27 m_{\tilde{g}}^{4} m_{\tilde{q}}^{2}-54 m_{\tilde{g}}^{2} m_{\tilde{q}}^{4}+18 m_{\tilde{q}}^{6}\right) \log \left(m_{\tilde{g}}^{2} / m_{\tilde{q}}^{2}\right)}{9\left(m_{\tilde{g}}^{2}-m_{\tilde{q}}^{2}\right)^{4}}
\end{aligned}
$$

We use these QCD dimension six operators in order to obtain the non-degeneracy bounds of left-right squark masses.

\section{References}

[1] N. Haba, K. Kaneta, S. Matsumoto, T. Nabeshima and S. Tsuno, Phys. Rev. D 85 (2012) 014007, arXiv:1109.5082 [hep-ph].

[2] S. Berge, W. Hollik, W. M. Mosle and D. Wackeroth, Phys. Rev. D 76 (2007) 034016, hep-ph/0703016.

[3] G. Aad et al. Atlas Collaboration, arXiv:1110.2299 [hep-ex].

[4] V. M. Abazov et al. , D0 Collaboration, Phys. Lett. B660 (2008) 449-457, arXiv:0712.3805[hep-ex].

[5] G. T. Bodwin, E. Braaten, G. P. Lepage, Phys. Rev. D51 (1995) 1125-1171, hep-ph/9407339.

[6] H. Kleinert, Phys. Lett. B62 (1976) 429.

[7] T. Kugo, Phys. Lett. B76 (1978) 625.

[8] T. Morozumi, H. So, RIFP-671 (1986). 
[9] J. Hisano, S. .Matsumoto, M. M. Nojiri, O. Saito, Phys. Rev. D71 (2005) 063528, hep-ph/0412403.

[10] N. Brambilla et al., Quarkonium Working Group Collaboration, hep-ph/0412158.

[11] N. Brambilla, A. Pineda, J. Soto, A. Vairo, Rev. Mod. Phys. 77 (2005) 1423, hep-ph/0410047.

[12] N. Brambilla, S. Eidelman, B. K. Heltsley, R. Vogt, G. T. Bodwin, E. Eichten, A. D. Frawley, A. B. Meyer et al., Eur. Phys. J. C71 (2011) 1534, arXiv:1010.5827 [hep-ph].

[13] E. Eichten, K. Gottfried, T. Kinoshita, K. D. Lane and T. -M. Yan, Phys. Rev. D 17 (1978) 3090 [Erratum-ibid. D 21 (1980) 313].

[14] M. J. Duncan, Nucl. Phys. B 214 (1983) 21.

[15] K. Nakamura et al., Particle Data Group Collaboration, J. Phys. G G37 (2010) 075021.

[16] G. Aad et al. [ATLAS Collaboration], arXiv:1206.1760 [hep-ex].

[17] S. Necco and R. Sommer, Phys. Lett. B 523 (2001) 135, hep-ph/0109093.

[18] S. Recksiegel and Y. Sumino, Phys. Rev. D 65 (2002) 054018, hep-ph/0109122.

[19] S. Recksiegel and Y. Sumino, Eur. Phys. J. C 31 (2003) 187, hep-ph/0212389.

[20] L. L. Foldy, S. A. Wouthuysen, Phys. Rev. 78 (1950) 29-36.

[21] S. Tani, Prog. Theor. Phys. 6 (1951) 267-285.

[22] H. D. Politzer, Nucl. Phys. B172 (1980) 349.

[23] N. Haba, K. Kaneta, S. Matsumoto, T. Nabeshima, arXiv:1106.6106[hep-ph]. 